\title{
LIOFILIZACIÓN DE PULPA DE Myrciaria dubia HBK MC VAUGH, CAMU CAMU
}

\author{
Rodney Vega Vizcarra*
}

\section{RESUMEN}

El Myrciaria dubia HBK Mc Vaugh, camu camu, es un fruto nativo de la Amazonía peruana, que se caracteriza por su alto contenido de ácido ascórbico, con valores promedio de 2780 mg (Instituto Nacional de Nutrición del Perú, 1996), 2994 mg (Villachica et al. 1996); en comparación con la acerola con 1300 mg (Villachica et al. 1998) y 1790 mg (Instituto Nacional de Nutrición del Perú, 1996; Instituto Nacional de Nutrición de Buenos Aires) en Riva \& Gonzales (1996); superando también a frutos cítricos como el limón, naranja y otros. La vitamina C o ácido ascórbico, se considera como antiescorbútica, potenciadora del sistema inmunológico y además, protege contra la gripe y resfriados.

Con esta especie se abre la posibilidad de utilizar una buena cantidad de frutos de camu camu producidos en la región, generando así industrias y trabajo en la región amazónica del Perú.

Sin embargo, este fruto, así como la vitamina C que contiene, son muy sensibles tanto al manipuleo como a los procesos de extracción de pulpa y almacenamiento, incrementado por el alto contenido de agua, por lo cual se busca producir pulpa deshidratada por procesos de liofilización. Estos procesos fueron efectuados utilizando pulpa de camu camu, a partir de frutos pintón maduro procedentes de la ciudad de Pucallpa, Perú.

Los procesos de liofilización fueron efectuados con pulpa de camu camu entera y sin concentrar, a temperaturas de $-40,-50$ y $-60{ }^{\circ} \mathrm{C}$, habiendo obtenido concentraciones de ácido ascórbico de hasta 20 383,80 y mejor coloración, con $-40{ }^{\circ} \mathrm{C}$. Los productos obtenidos a -50 y $-60^{\circ} \mathrm{C}$, presentan menores concentraciones de ácido ascórbico, variando de color hasta un rosado anaranjado.

Palabras clave: bionegocios, liofilización, camu camu deshidratado, valor agregado.

\begin{abstract}
ABSTACT
The Myrciaria dubia HBK Mc Vaugh, camu camu, a native fruit of the Peruvian Amazonia, is characterized by its high content of ascorbic acid, $2780 \mathrm{mg}$. (National Institute of Nutrition of the Peru, 1996), $2994 \mathrm{mg}$ (Villachica et all. 1996), in comparison with, acerola, $1300 \mathrm{mg}$ (Villachica et all. 1998) and $1790 \mathrm{mg}$ (National Institute of Nutrition of Peru, 1996; National Institute of Nutrition of Buenos Aires), in Riva \& Gonzales (1996). Also, overcoming to citric fruits as the lemon, orange, and others. The vitamin $\mathrm{C}$ or ascorbic acid is considered anti scorbutic, immunologic system enhancer, protection against flu and colds and it opens the possibility to use a whole production of camu camu fruits in this region, generating jobs in this Amazon region of the Peru.
\end{abstract}

However, this fruit, and the vitamin C that contains, is very sensible, while cropping, handling, processing pulp and storage, increased by the high content of water in the fruit. That is why this experiment expects to produce dehydrated fruit pulp by freeze dried (lyophilization) processes. These freeze dried processes were made using raw pulp of camu camu, obtained from close mature fruits cropped from the city of Pucallpa, Ucayali region, Perú.

The freeze dried processes were made up of without concentrating whole pulp of camu camu, using temperatures of $-40,-50$ and $-60^{\circ} \mathrm{C}$, having obtained bigger concentrations of acid ascorbic up to 20383,80 and better coloration at $-40^{\circ} \mathrm{C}$. The products obtained at -50 and $-60^{\circ} \mathrm{C}$, present smaller concentrations of ascorbic acid than $40^{\circ} \mathrm{C}$.

Key words: biobussines, lyophilization, camu camu dehydrated, added value.

* Investigador del IIAP-Ucayali 


\section{INTRODUCCIÓN}

El camu camu está considerado como un cultivo con gran potencial para lograr valor agregado de alto nivel, especialmente por el alto contenido de ácido ascórbico, 2780 mg según Bejarano \& Bravo (1990), siendo hasta el momento el único fruto que se encontró con esa cantidad, sobrepasando muchas veces los $3000 \mathrm{mg} / 100 \mathrm{~g}$ de pulpa fresca (Pinedo et al. 2001).

Los análisis bromatológicos muestran cantidades bastante altas de ácido ascórbico reducido (2880 mg/100 g) y ácido ascórbico total (2994 mg/100 g) (Cuadro 1). Estos valores son 63 veces más grandes que los encontrados en jugo de limón y 2,1 veces mayores que los encontrados en la acerola. También son importantes los contenidos de aminoácidos esenciales como valina y leucina, los que son muy necesarios principalmente para el desarrollo órgano funcional en la etapa infantil (Robinson 1991).

Para fines industriales, es preferible contar con frutos en estado pintón maduro, ya que soportan mejor los procesos previos a la transformación, pero para lograr un color rosa más acentuado en la pulpa obtenida, se cosechan los frutos en estado maduro (Vega 2002).

El desarrollo de valor agregado está relacionado con la maduración de los frutos, variando para una misma planta desde verde (0,0 \% coloración granate), verde pintón (25-50\% coloración granate), pintón (50-75 coloración granate) y maduro (75-100\% coloración granate) (Imán 2000), (Pinedo et al. 2001), (Riva \& Gonzales 1997). Por otra parte, Vásquez (2000) indica que actualmente es posible obtener pulpa congelada, concentrada, deshidratada, atomizada. Pero también puede obtenerse pulpa refinada, liofilizada, atomizada (Vega 2001) y productos con alto valor agregado como sachets, polvos hidrolizables, complementos vitamínicos, entre otros (Vega 2000).

Estos procesos son muy importantes para incrementar el contenido vitamínico de la pulpa del camu camu, así como para conservar la vitamina $\mathrm{C}$ por el mayor tiempo posible sin sufrir mayor disminución, ya que es considerada la más sensible y lábil, susceptible de deteriorarse fácilmente por oxidación, cambios de pH, temperatura y acción de la luz, entre otros (Belitz-Grosch 1985).

Entre estos, uno de los procesos más adecuados es la liofilización, que genera la deshidratación por congelación y sublimación, bajo condiciones cuidadosamente controladas de presión y temperatura, para dejar una estructura que revierta el estado previo, por adición de agua (Amos 1986).

El objetivo del presente trabajo fue obtener pulpa pura y entera deshidratada de camu camu, por proceso de liofilización y determinar los contenidos de ácido ascórbico. Mediante este proceso se busca eliminar el contenido de agua presente en la pulpa de camu camu. 


\section{MATERIAL Y MÉTODO}

La materia prima utilizada fue pulpa de camu camu extraída a partir de frutos en estado de maduración pintón maduro, procedentes de la estación experimental Pacacocha, INIA Pucallpa, con una concentración inicial de 2050 mg \% de ácido ascórbico.

La pulpa de los frutos cosechados de camu camu al estado pintón maduro, fue extraída en una pulpeadora de acero inoxidable de $250 \mathrm{~kg} / \mathrm{h}$ de capacidad nominal, con paletas modificadas y a $1800 \mathrm{rpm}$. La concentración de sólidos totales de la pulpa fue determinada con refractómetro manual con escala de 0-32 grados Brix ( ${ }^{\circ} \mathrm{Bx}$ ).

La materia prima seleccionada fue pulpa de camu camu obtenida a partir de frutos en estado de maduración pintón maduro; ésta fue uniformizada para obtener la muestra con un contenido de ácido ascórbico de $2050 \mathrm{mg}$ \% y 6,0 Brix, de coloración rosada rojiza y un contenido de humedad del 93\%.

La temperatura de la pulpa fue registrada con un termómetro digital con vástago de acero inoxidable con escala de $-50 \mathrm{a}+200^{\circ} \mathrm{C}$. Esta pulpa fue envasada en bolsas de polietileno de media densidad, color transparente y capacidad de un kg. Las muestras fueron pesadas en una balanza gramera con escala de 0 a $2000 \mathrm{~g}$. La pulpa así envasada, fue almacenada en congeladores a temperaturas de $-20^{\circ} \mathrm{C}$ y transportada a los centros de procesamiento en cajas de Tecnoport debidamente selladas.

La pulpa fue descongelada para ser distribuida en las bandejas del liofilizador y ser nuevamente congelada hasta una temperatura de $-40^{\circ} \mathrm{C}$. El proceso fue efectuado en un liofilizador de tamaño piloto, usando amoniaco como refrigerante y ocho mbars de vacío. Fueron efectuados procesos a $-40,-50$ y $-60{ }^{\circ} \mathrm{C}$. Los procesos duran en promedio 24 horas.

El producto obtenido, fue un producto seco, granulado, de color rosado intenso y que no necesita ser molido. El envasado se efectuó en bolsas de polietileno de color azul oscuro, selladas herméticamente para luego almacenarlas a temperatura de congelamiento $-20^{\circ} \mathrm{C}$.

Los análisis del pH de las muestras fueron efectuados utilizando el método 925.22 de la AOAC (1995). Para determinar los ÚBx, fue utilizado un refractómetro con prisma de cuarzo, escala 0-32, y se usó el método 932.12 de la AOAC (1995). Para determinar el contenido de humedad se utilizo el método 934.06 de la AOAC (1995), así como para determinar el contenido de ácido ascórbico, fue utilizado el método de titulación con el 2.6diclorofenolindofenol.

\section{RESULTADOS Y DISCUSIÓN}

De las tres temperaturas a las cuales fue efectuada la liofilización, se obtuvieron mejores características organolépticas y sobre todo mayor concentración de ácido ascórbico con la temperatura de $-40{ }^{\circ} \mathrm{C}$, como se observa en la tabla 1. 
Tabla 1. Concentraciones de ácido ascórbico en dos procesos de liofilizado a diferentes temperaturas

\begin{tabular}{|c|c|c|}
\hline${ }^{\circ} \mathrm{C}$ & $1^{\text {er }}$ proc. & $2^{\text {do }}$ proc. \\
\hline $\mathbf{- 4 0}$ & $15838.70(\mathrm{a})$ & $20383.80(\mathrm{a})$ \\
\hline $\mathbf{- 5 0}$ & 16833.17 & 19960.74 \\
\hline $\mathbf{6 0}$ & 15849.73 & 19855.23 \\
\hline
\end{tabular}

Como se puede observar en la tabla 1 , la pulpa liofilizada a $-40^{\circ} \mathrm{C}$, presenta las mayores cantidades de ácido ascórbico, especialmente en el segundo proceso de liofilizado, durante en cual se tuvo especial cuidado en las determinaciones de esta vitamina.

Asimismo, las características organolépticas de la pulpa liofilizada, son muy similares en color, aroma, olor y sabor a los de la fruta natural de la cual se obtuvo la pulpa, habiendo concentrado su contenido de ácido ascórbico en 7,72 y 9,94 veces para el primer y segundo procesos, respectivamente.

En la figura 1, se observa que en el $1^{\mathrm{er}}$ proceso $(1 \mathrm{~L})$, existe una cantidad ligeramente mayor de ácido ascórbico en la muestra a $-50^{\circ} \mathrm{C}$ (LF-50), siguiendo con la de $-60^{\circ} \mathrm{C}$ (LF-60) y la de $-40^{\circ} \mathrm{C}$ (LF-40). En el $2^{\circ}$ proceso (2L), la pulpa obtenida a $-40^{\circ} \mathrm{C}$ (LF-40), contiene mayor cantidad de ácido ascórbico, seguida por la de $-50^{\circ} \mathrm{C}$ (LF-50) y $-60^{\circ} \mathrm{C}$ (LF-60).

Sin embargo, luego del análisis estadístico SAS-Duncan, no se ha observado diferencia entre los tratamientos LF-40, LF-50 y LF-60 al 5\% de significancia, para cada uno de los procesos respectivamente.

Los productos generados en estos procesos también están muy relacionados con las características organolépticas del producto liofilizado obtenido, principalmente con el color y aroma, porque la que más conserva el color de la pulpa original, es el LF-40, seguido del procesado LF-50 y por el LF-60.

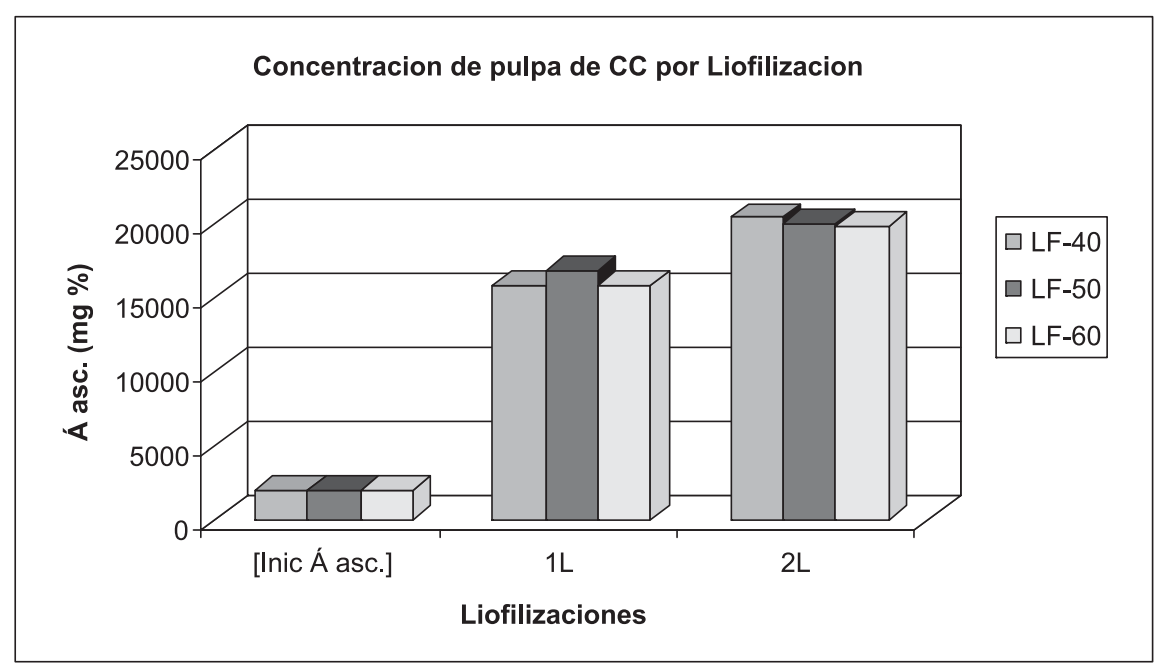

Figura 1. Niveles de ácido ascórbico en los procesos de liofilización. 
Esta figura muestra también el incremento del contenido de ácido ascórbico desde una cantidad inicial de 2050 mg \% presente en la pulpa, hasta $20383,80 \mathrm{mg} \%$. Esta cantidad se mantiene casi constante durante el tiempo de almacenamiento, como se muestra en la figura 2.

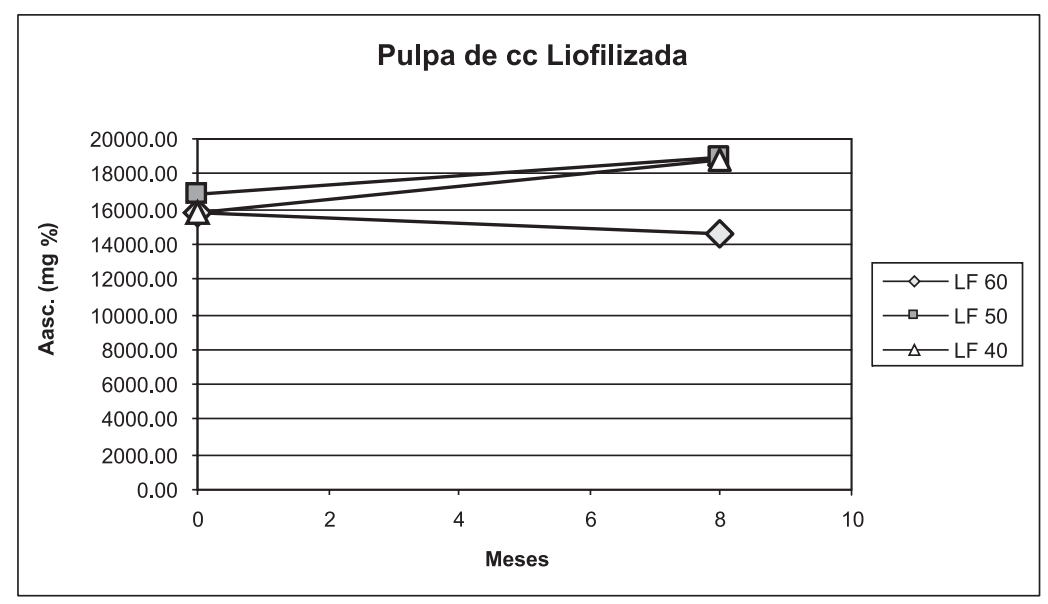

Figura 2. Variación del contenido de ácido ascórbico durante el almacenamiento.

La figura 2, muestra la variación del contenido de ácido ascórbico, durante un tiempo de almacenamiento de ocho meses a $-20^{\circ} \mathrm{C}$, en envases de PVC color negro. Se observa que los procesos LF-40 y LF-50 muestran un aparente incremento de la concentración de ácido ascórbico luego de ocho meses, lo cual podría ser causado por la mayor disponibilidad o liberación de más vitamina al medio en el tiempo; por la falta de tensión superficial a falta de agua, sin embargo, contrariamente se observa que en el caso del tratamiento LF-60, se presenta una ligera disminución hasta el octavo mes.

\section{CONCLUSIONES Y RECOMENDACIONES}

- $\quad$ El producto liofilizado con mayor concentración de ácido ascórbico, se encontró en la muestra LF-40.

- $\quad$ Los procesos para las muestras LF-50 y LF-60, presentan concentraciones menores que en LF-40. Sin embargo se observa que generan pequeñas variaciones en el color del producto.

- Luego del análisis estadístico no se ha observado diferencias entre los tratamientos LF-40, LF-50 y LF60 al 5\% de significancia, para cada uno de los procesos de liofilizado 1L y 2L respectivamente.

- $\quad$ El producto liofilizado de pulpa entera de camu camu, no necesita ser sometido a molienda al final del proceso.

- $\quad$ La cantidad final de humedad del producto fue de $2 \%$ en promedio.

- $\quad$ Se deben profundizar más los estudios, principalmente utilizando pulpa de camu camu en los diversos estadios de maduración y de diferentes procedencias.

- $\quad$ Se debe probar la separación de la fibra de la pulpa de camu camu antes del proceso de liofilización. 


\section{BIBLIOGRAFÍA}

AMOS, A. J. 1968. Manual de industrias alimentarias. Ed. Acribia. Zaragoza.

AOAC. 1995. Official methods of analysis. Agricultural Chemicals Contaminants and Drugs. $15^{\circ}$ Ed. Gaithersburg, Md. USA.

BEJARANO, E. y M. BRAVO, et al. 1990. Tabla de composición de alimentos industrializados. Ministerio de Salud, Instituto Nacional de Nutrición, Lima-Perú.

BELITZ-GROSCH. 1985. Química de los alimentos. Acriba. Zaragoza.

CORREA, I. 2000. Cultivo de camu camu Myrciaria dubia H.B.K. en la región Loreto. Dirección de Transferencia de Tecnología Agraria-INIA. Lima-Perú.

PINEDO, M., et al. 2001. Sistema de producción del camu camu en restinga. Manual Técnico IIAP. Iquitos-Perú.

RIVA, R. e I. GONZALES. 1996. Tecnología del cultivo de camu camu en la Amazonía peruana. Editorial INIA. Pucallpa-Perú.

ROBINSON, J. 1991. Bioquímica y valor nutritivo de los alimentos. Editorial Acriba. Zaragoza.

ROCA, N. A. 1965. Estudio químico bromatológico de la Myrciaria paraensis Berg, su contenido de ácido ascórbico. Tesis para optar al titulo de químico, Universidad Nacional Mayor de San Marcos (UNMSM). Lima-Perú.

VÁSQUEZ, M. A. 2000. El camu camu; cultivo, manejo e investigaciones. Editora Gráfica e Imprenta Universal SRL. Iquitos-Perú.

VEGA, V. R. 2000. Valor agregado en camu camu. Informe final de investigación, IIAP Pucallpa, Perú.

VEGA, V. R. 2001. Valor agregado en camu camu. Informe final de investigación, IIAP Pucallpa, Perú.

VEGA, V. R. 2002. Valor agregado en camu camu. Informe final de investigación, IIAP Pucallpa, Perú.

VILLACHICA, H. 1996. Frutales y hortalizas promisorias de la Amazonía. Tratado de Cooperación Amazónica TCA. Lima-Perú. 


\title{
MICORRIZAS ARBUSCULARES EN ULTISOLES DE LAAMAZONÍA PERUANA
}

\author{
ARBUSCULAR MYCORRHIZAL IN ULTISOLS IN THE PERUVIANAMAZONIA
}

\author{
Pedro O. Ruiz ${ }^{1}$ y Charles B. Davey ${ }^{2}$
}

\section{RESUMEN}

Se evaluaron los niveles de colonización micorrícica y la morfología de los hongos que forman micorrizas arbusculares (MA) en especies de plantas en las siguientes opciones de manejo de suelos: 1) barbecho de bosque secundario de 15 años, 2) barbecho de bosque secundario de 5 años, 3) sistema de producción en multiestrato, 4) plantación de Bactris gasipaes, pijuayo, 5) sistemas de cultivos continuos con bajos insumos y 6) sistema de cultivos continuos con altos insumos. Se encontraron altos niveles de colonización en la mayoría de árboles (> 70\%). Algunos árboles con pelos radiculares largos tuvieron niveles más bajos de colonización micorrícica que en aquellos sin estas estructuras radiculares. En algunos árboles, la colonización micorrícica aumentaba con el incremento de arcilla en el suelo. Los cultivos anuales tuvieron niveles de colonización micorrícica más bajos que la mayoría de árboles. Las estructuras morfológicas de los hongos MA observadas en las raíces de las especies evaluadas fueron altamente variables. Se podrían establecer asociaciones preferenciales de hongos MA/plantas hospederas, mediante la detección en la raíces de las plantas de algunas estructuras morfológicas inherentes a una especie de hongo MA en particular. Las especies de hongos MA pueden ser afectadas por el manejo del suelo. Especies de Glomus parecen tolerar rangos más amplios de acidez del suelo que especies de Acaulospora, las que están más restringidas a suelos ácidos. Especies de Gigaspora y Scutellospora fueron las menos predominantes en los suelos estudiados.

Palabras clave: micorrizas arbusculares, hongos, manejo de suelos.

\begin{abstract}
Levels of arbuscular mycorrhizal colonization and the morphology of arbuscular mycorrhizal (AM) fungi were evaluated in plant species in the following soil management options: 1) a 16-year-old secondary forest fallow, 2) a 5-year-old secondary forest fallow, 3) a multistrata tree-crop production system, 4) a peach palm (Bactris gasipaes) plantation, 5) a low-input continuous cropping system, and 6) a high-input continuous cropping system. High levels of mycorrhizal colonization (> 70\%) were found in most trees. Some trees with long root hairs had lower mycorrhizal colonization levels than trees lacking these root structures. In some trees, mycorrhizal colonization increases with increasing clay contents in soil. Annual crops had lower mycorrhizal colonization levels than most trees. Morphological structures of AM fungi observed in roots of plant species evaluated were highly variable. Preferential AM fungi / host plant associations might be established by detecting in plant roost some mycorrhizal fungi morphological structures inherent to a particular fungal genus. Species of AM fungi seem to be affected by soil management. Glomus sp. Appears to tolerate wider ranges of soil acidity than Acaulospora sp. Which is more restricted to acid soils. Gigaspora sp. And Scutellospora sp. Were the less predominant genera of AM fungi in the soil management system studied.
\end{abstract}

Key words: arbuscular mycorrhizal, fung, soil management.

\section{INTRODUCCIÓN}

En los bosques amazónicos, así como en la mayoría de áreas en los trópicos bajos húmedos, las raíces de las plantas están asociadas con hongos que forman micorrizas arbusculares (MA), especialmente las especies arbóreas (Janos 1980; St. John 1980a 1980b; Ruiz et al. 1989. Su prevalencia sugiere que las micorrizas pueden ser

1 Consultor. AndeStudio S.A. Manejo de suelos, revegetación, micorrizas. Correo electrónico: interandes@terra.com.pe

2 Professor Emeritus. Soil Science and Forestry Departments. North Carolina State University. Raleigh, N.C., U.S.A. 
de gran importancia para el crecimiento de las plantas en suelos deficientes en fósforo, tal como los ultisoles de la Amazonía (Salinas \& Sánchez 1976).

Un número de plantas, sin embargo, dependen más de las micorrizas para obtener fósforo que otras, y esto ha sido relacionado ya sea al metabolismo de fosfatos o a la estructura de la raíz (Baylis 1975; St. John 1980c; Fitter 1989).

Por otro lado, los porcentajes de colonización en las raíces, no indican con seguridad la dependencia de las micorrizas (Janos 1987) y la extensión de la dependencia a las micorrizas no establece la magnitud del beneficio a la planta o la efectividad de la asociación (Lópes \& Siquiera 1980). En algunos casos, las mismas especies de hongos MA pueden ser efectivas y una sola especie hospedera y patogénica en otra (Crush 1976).

Los suelos característicamente contienen más de una especie de hongo MA y aunque muchas de éstas parecen ser cosmopolitas, algunas pueden ser estrictamente tropicales, por ejemplo, Acaulospora tuberculata y A. foveata (Janos \& Trappe 1982; Nicolson \& Schenck 1979).

Mientras la infección preferencial no está detectada comúnmente en condiciones de laboratorio o invernadero, usando un número de organismos experimentales, puede ocurrir en condiciones naturales, cuando la planta queda expuesta a una mayor diversidad de poblaciones microbianas en su ambiente natural.

Se desconoce la importancia de la especificidad durante la colonización (capacidad de colonización) o durante el crecimiento de la planta (efectividad) en la habilidad competitiva de la planta y en la estructura de la comunidad florística. Los métodos utilizados para evaluar las micorrizas no hacen distinciones entre hongos en las raíces colonizadas.

La morfología de los hongos MA, sin embargo, puede cambiar en la raíces de diferentes hospederos y aún en raíces de diferentes edades en una misma planta (Abbott \& Robson 1999ª). Algunas características edáficas como la provisión de fósforo, pueden llevar a alteraciones en la morfología de los hongos MA en las raíces (Mosse 1973). La identificación de los hongos MA al nivel de especie requiere de microscopios de alta resolución y probablemente de procedimientos sofisticados como electroforesis, y técnicas serológicas y moleculares, las que no están disponibles en la mayoría de laboratorios en los trópicos.

Algunas características morfológicas de los hongos MA, como esporas con sus hifas de subtensión, vesícula, arbúsculos, células auxiliares, desarrollo de hifas tipo Paris y Arum (Smith \& Smith 1997), etc., reconocibles bajo un microscopio de baja resolución, permiten su identificación, por lo menos a nivel de género.

Adicionalmente, las prácticas agrícolas modifican las propiedades físicas, químicas y biológicas, las que pueden alterar la habilidad de los hongos MA para colonizar raíces (Abbott \& Robson 1991a). Prácticas agrícolas como el encalado y las aplicaciones de fosfato y el cambio de especies de plantas pueden alterar asimismo, la dominancia de especies de hongos MA (Abbott \& Robson 1991b).

La concurrencia de micorrizas en los diferentes ecosistemas ha sido objeto de numerosas investigaciones que han demostrado su importancia en muchos lugares.

Sin embargo, existe la necedad de más investigación basada en observaciones de campo en cuanto a las relaciones de las especies de hongos MA y la composición florística en los trópicos húmedos. Este tipo de estudio puede parecer de poco uso práctico, pero inevitablemente será de gran beneficio para la investigación forestal, agroforestal, recuperación de suelos, etc. y además nos ayudará a entender el funcionamiento de las micorrizas arbusculares en los ecosistemas de bosque húmedo tropical.

Los objetivos principales del presente estudio fueron: evaluar al grado de infección micorrícica arbuscular, determinan las diferencias en la morfología de los hongos MA en las raíces de especies de plantas e identificar las especies de hongos MA en ultisoles bajo diferentes prácticas de manejo en la cuenca amazónica del Perú. 


\section{MATERIAL Y MÉTODO}

El estudio fue conducido en la Estación Experimental de Yurimaguas, ubicada en la cuenca amazónica del Perú ( $76^{\circ} 05^{\prime} \mathrm{W}, 05^{\circ} 45^{\prime} \mathrm{S}$ a $180 \mathrm{msnm}$ ). El área tiene un promedio anual de lluvia de $2200 \mathrm{~mm}$ y una temperatura promedio anual de $26^{\circ} \mathrm{C}$.

El área utilizada está localizada en un paleudult típico, fino-limoso, silíceo, isohipertémico, con pendientes que van de $0-5 \%$. La vegetación corresponde a un barbecho de bosque secundario de 10 años. El experimento consistió de seis opciones de manejo como tratamientos con tres repeticiones distribuidas en un diseño de bloques completamente randomizados, con parcelas de $1875 \mathrm{~m}^{2}(75$ x $25 \mathrm{~m})$. Los tratamientos fueron dispuestos en bloques de acuerdo a la textura del suelo. Los bloques 1 y 3 con una textura en la capa superficial de suelo arenosa-franca ( 7 y $8 \%$ de arcilla, respectivamente), y el bloque 2 con una textura franco-arenosa (17\% de arcilla). El suelo tiene un valor de $\mathrm{pH}$ de 4.6, $10 \mathrm{ppm}$ de fósforo (extracto Olsen modificado), $1.8 \mathrm{cmol}_{\mathrm{c}} \mathrm{L}^{-1} \mathrm{de}$ acidez intercambiable, 0.81 de calcio, 0.16 de magnesio y 0.10 de potasio, y una saturación de aluminio de $36 \%$ (ver la tabla 1 para el análisis realizado en 1991).

Tratamientos: barbecho de bosque testigo. Estas parcelas fueron cercadas y permanecen sin ser intervenidas.

Se realizó el muestreo de raíces para la evaluación de infección micorrícica siguiendo la metodología del Tropical Soil Biology and Fertility-TSBF (1992) para la caracterización del sitio. Muestras de raíces fueron colectadas a los $10 \mathrm{~cm}$ superficiales del suelo durante agosto de 1991 (estación seca) para evaluar la infección micorrícica. En los bosques secundarios de 6 a 15 años, se evaluaron solamente las especies arbóreas, colectándose las raíces de cinco posiciones, cada una de $30 \mathrm{~cm}$ del árbol, las que fueron agrupadas en una sola masa. Para los componentes arbóreos en el sistema en multiestrato y en la plantación de pijuayo, la colección de raíces fue similar que en los bosques; las leguminosas de cobertura fueron también colectadas en cinco posiciones en cada parcela y luego agrupadas en una sola. La colección de raíces en los tratamientos de altos y bajos insumos se realizó en cinco pociones dentro de cada parcela y luego agrupadas en una. En la estación lluviosa (diciembre de 1992), el muestro de raíces en los dos tratamientos de bosque secundario se hizo solamente en las especies presentes en las tres repeticiones. Para los otros tratamientos, la colección de raíces fue similar que en la estación seca.

Tabla 1. Características químicas de los suelos (0-15 cm profundidad) bajo diferentes sistemas de manejo en Yurimaguas, Perú.

\begin{tabular}{|c|c|c|c|c|c|c|c|c|}
\hline \multirow[t]{2}{*}{ Tratamiento } & Acidez & $\mathbf{P}$ & $\begin{array}{c}\text { Acidez } \\
\text { intercamb. }\end{array}$ & Ca & Mg & $\mathbf{K}$ & CIC & \multirow{2}{*}{$\begin{array}{c}\text { Sat } \\
\text { Al }\end{array}$} \\
\hline & $\mathbf{p H}$ & $\mathbf{M g} / \mathrm{L}$ & \multicolumn{5}{|c|}{$\operatorname{cmol}(+) / L$} & \\
\hline Bosques sec. 5 años & 4.8 & 7.0 & 0.36 & 0.9 & 0.23 & 0.11 & 1.63 & 21.5 \\
\hline Cult. cont. altos insumos & 5.8 & 27.4 & 0.07 & 1.7 & 0.65 & 0.16 & 2.66 & 1.8 \\
\hline Cult. cont bajos insumos & 4.8 & 12.0 & 0.60 & 0.7 & 0.14 & 0.09 & 1.59 & 32.4 \\
\hline Multiestrato & 4.6 & 5.0 & 0.53 & 0.7 & 0.14 & 0.07 & 1.41 & 29.9 \\
\hline Plantación pijuayo & 4.5 & 5.0 & 0.33 & 0.7 & 0.11 & 0.10 & 1.27 & 20.9 \\
\hline Bosques sec. 16 años & 4.6 & 6.4 & 1.13 & 0.7 & 0.19 & 0.11 & 2.17 & 47.0 \\
\hline
\end{tabular}


Las raíces fueron clareadas en $\mathrm{KOH}$ caliente al 10\% y teñidas con azul de tripano en una solución de glicerina, ácido láctico y agua (Phillips \& Hayman 1970; Shafer, comunicación personal). Este método fue modificado, eliminando el uso de fenol y de ácido clorhídrico y utilizando un autoclave durante seis minutos para el proceso de clareamiento y teñido. Estas modificaciones ahorran tiempo y adicionalmente son seguras, ya que el fenol y el HCI son compuestos altamente tóxicos. La infección micorrícica se midió mediante el método de intersecciones propuesto por Giovanetti \& Mosse (1980).

Las características morfológicas de los hongos MA presentes en las raíces fueron también evaluadas con la intención de distinguir diferencias en las poblaciones de hongos. Se preparó una clave para la identificación de las distintas estructuras de estos hongos.

Adicionalmente, se tomaron muestras de suelo de las mismas parcelas para evaluar la diversidad de poblaciones de hongos MA. Se tomaron 50 gr de suelo, los que fueron tamizados y decantados en húmedo para recuperar esporas, las que fueron separadas de acuerdo al género y luego contadas utilizando un microscopio estereoscópico a 60 aumentos (Gerdemann \& Nicolson 1963). Los valores expresados como número total de esporas por 100 gr de suelo seco. La esporas se montaron con PVL (alcohol polivinilo) en placas porta objeto de vidrio e identificadas a nivel de género.

Los datos de infección micorrícica para los tratamientos donde las especies de plantas estuvieron presentes en las tres repeticiones durante las dos estaciones, fueron analizadas estadísticamente mediante el procedimiento ANOVA (Statistical Analysis System 1985). Para comparación de promedios se utilizó un nivel de significación de $\mathrm{p}<0.05$ (a menos que se especifique de otra manera).

\section{RESULTADOS Y DICUSIÓN}

En la primera evaluación correspondiente a la estación seca, se examinaron la mayoría de especies en los barbechos de bosque secundario de 5 y 16 años. Para la segunda evaluación, correspondiente a la estación lluviosa, se examinaron solamente las especies presentes en las tres repeticiones. Los resultados y la discusión del barbecho de bosque secundario de 16 años se presentan primero, como una línea base para compararlos con los otros tratamientos en estudio.

\section{Colonización micorrícica}

Barbecho de bosque secundario de 16 años

La composición florística en este barbecho de bosque fue diversa, considerando el área evaluada (1875 m²). Se encontraron un total de 31 especies correspondientes a 23 familias (aproximadamente 165 especies de árboles por hectárea). Se estableció un récord mundial en 1988 por A.H. Gentry, quien identificó cerca de 300 especies de árboles en cada uno de dos parcelas de una hectárea cerca de Iquitos, en la Amazonía del Perú (Wilson 1989).

Todos los árboles evaluados estaban asociados con hongos MA (tabla 2). Miembros de las familias Annonaceae, Bignonaceae, Malastomaceae, Piperaceae, Monimiaceae, Apocynaceae, Lauraceae, Guttiferae, Meliaceae y Bombacaceae presentaron un alto grado de infección micorrícica $(<75 \%)$. Entre las leguminosas, miembros de las familias Fabaceae y de la subfamilia Mimosaceae también presentaron altos niveles de infección, excepto miembros de la subfamilia Caeesalpinaceae. Se encontró una amplia variación en miembros de las familias Moraceae. Por otro lado, miembros de las familias Rubiaceae, Ulmaceae, Myristicaceae, Elaoecarpaceae y Melastomaceae presentaron niveles moderados y ligeros de infección mocorrícica (de 70 a 31\% respectivamente). 
Tabla 2. Colonización micorrícica en las especies del barbecho de bosques de 16 años. Estación seca (agosto, 1991).

\begin{tabular}{llc}
\hline Nombre vernacular* & Nombre científico & Micorriza (\%) \\
\hline Anacaspi & Cynometra sp. (Caesalpinaceae) & 25 \\
Cetico & Cecropia sp. (Moraceae) & 41 \\
Huarmi huarmi & Compsoneura capitellata (Myristicaceae) & 61 \\
Chontaquiro & Diplotropis martiusii (Fabaceae) & 62 \\
Yanahuasca & Guatteria sp. (Annonaceae) & 76 \\
Huangana caspi & Sloanea aff. megaphylla (Elaeocarpaceae) & 62 \\
Ishtapi & Jacaranda copaia (Bignonaceae) & 91 \\
Hornopichana & & 37 \\
Icoja & Unonopsis floribunda (Annonaceae) & 79 \\
Cumala blanca & Virola flexuosa (Myristicaceae) & 31 \\
Pichirina & Vismia sp. (Guttiferae) & 90 \\
Yacushapana & Terminalia sp. (Combretaceae) & 70 \\
Acero caspi & Cosmibuena grandiflora (Rubiaceae) & 50 \\
Barbasco caspi & Lonchocarpus sp. (Fabaceae) & 85 \\
Níspero & Bellucia grossularoides (Melastomaceae) & 97 \\
Cordoncillo & Piper sp. (Piperaceae) & 82 \\
Uvilla & Pourouma cecropiifolia & 87 \\
Ojé & Ficus anthelmintica (Moraceae) & 83 \\
Pichohuayo & Siparuna sp. (Moinimiaceae) & 95 \\
Moena & Aniba sp. (Lauraceae) & 71 \\
Cumala colorada & Iryanthera sp. (Myristicaceae) & 60 \\
Shimbillo & Inga sp. (Mimosaceae) & 74 \\
Caracha caspi & Miconia petioilaris (Melastomaceae) & 69 \\
Cedromash & Cabralea canjerana (Meliaceae) & \\
Ciprana & & \\
\hline & & \\
\hline
\end{tabular}

* Lao (1986), Vásquez (1989), Vásquez \& Gentry (1989), Pinedo-Vásquez \& Chota-Inuma (1990), Phillips et al. (1994)

En este barbecho de bosques, solamente cuatro especies de árboles estuvieron presentes en las tres repeticiones: Jacaranda copaia, Vismia tomentosa, Cecropia sp. y Ciprana (tabla 3). Se encontraron diferencias significativas ( $\mathrm{p}=0.04)$ en infección micorríca entre estas especies. Cecropia sp. alcanzó el nivel más bajo. Para J. copaia, V. tomentosa y ciprana, la infección fue ligeramente más alta en la estación húmeda que en la seca. Para Cecropia sp. ocurrió lo contrario. Las diferencias en infección parecen que están relacionadas con la morfología de la raíz. Se encontró que Cecropía sp. tiene pelos radiculares largos, mientras que en las otras especies esta característica no estaba presente. 
Tabla 3. Colonización micorrícica del barbecho de bosque secundario de 16 años. Estación seca (agosto, 1991) y estación húmeda (diciembre, 1992). Los números entre paréntesis son las desviaciones estándar.

\begin{tabular}{|l|c|c|}
\hline \multirow{2}{*}{ Especie } & \multicolumn{2}{|c|}{ Colonización micorrícica (\%) } \\
\cline { 2 - 3 } & Estación seca & Estación húmeda \\
\hline Jacaranda copaia & $86(9.9)$ & $91(5.3)$ \\
Vismia tomentosa & $76(15.7)$ & $80(2.1)$ \\
Cecropia sp. & $60(26.2)$ & $40(11.6)$ \\
Ciprana & $62(19.5)$ & $87(5.5)$ \\
\hline $\mathrm{n}=3$ &
\end{tabular}

Las diferencias de infección micorrícica entre las estaciones seca y lluviosa no fueron significativas. La relativamente pequeña variación de la temperatura del suelo (régimen de temperatura del suelo isohipertérmico) y la carencia de estacionalidad en la precipitación (régimen de humedad del suelo údico) en la Amazonía (Sánchez 1976), en adición a una composición florística más estabilizada en este barbecho de bosque, parecen que han contribuido a la pequeña diferencia en infección micorrícica entre las dos estaciones.

Barbecho de bosque secundario de 5 años

La composición florística de este barbecho joven, originalmente un sistema de agricultura migratoria, fue también diversa. Se evaluaron un total de 29 especies pertenecientes a 23 familias.

Todos los árboles examinados estaban infectados con micorrizas (tabla 4). Esta situación fue constante en algunas especies y variada en otras. Niveles altos de infección (> 75\%) se encontraron en miembros de las Annonaceae, Apocinaceae, Bignonaceae, Bombacaceae, Boraginaceae, Flacourtiaceae, Monimiaceae, Piperaceae, Rubiaceae, Sterculiaceae y Ulmaceae. Similarmente representantes de la familia Fabaceae y subfamilia Mimosaceae presentaron niveles consistentemente altos de infección de micorrícica, a excepción de Cynometra, de la subfamilia Caesalpinaceae. Una amplia variación se observó en las Moraceae, Nyctaginaceae y Tiliaceae. Niveles ligeros de infección se encontraron en miembros de las familias Euphorbiaceae, Guttiferae, Meliaceae y Solanaceae.

Tabla 4. Colonización micorrícica en las especies del barbecho de bosque secundario de cinco años, estación seca (agosto 1991).

\begin{tabular}{llc}
\hline Nombre vernacular* & Nombre científico & Micorriza (\%) \\
\hline Llausaquiro & Heliocarpus popayanensis (Tiliaceae) & 34 \\
Ojé & Ficus anthelmintica (Moraceae) & 90 \\
Sachacetico & Pourouma caussopoa (Moraceae) & 66 \\
Shimbillo & Inga sp. (Mimosaceae) & 74 \\
Yanavara & Guatteria sp. (Annonaceae) & 88 \\
Anacaspi & Cynometra sp. (Caesalpinaceae) & 41 \\
Pichohuayo & Siparuna sp. (Monimiaceae) & 95 \\
Topa & Ochroma lagopus (Bombacaceae) & 94 \\
Siucahuito & Solanum hazenii (Solanaceae) & 70 \\
Tamborhuayo & & 85 \\
Cordoncillo & Piper sp. (Piperaceae) & 90 \\
Cuchara caspi & Ambelania occidentalis (Apocynaceae) & 82 \\
Icoja & Unonopsis floribunda (Annonaceae) & 85 \\
Azarsisa & & 97 \\
Cetico & Cecropia sp. (Moraceae) & 93
\end{tabular}




\begin{tabular}{lll} 
Barbasco & Lonchocarpus utilis (Fabaceae) & 94 \\
Sacha cacao & Theobroma sp. (Sterculiaceae) & 75 \\
Ubiamba & & 95 \\
Mojara caspi & Alchornea spp. (Euphorbiaceae) & 70 \\
Atadijo & Trema micranta (Ulmaceae) & 98 \\
Tornillo & Cedrelinga catanaeformis (Mimosaceae) & 85 \\
Guaba & Inga sp. (Mimosaceae) & 90 \\
Ishatapi & Jacaranda copaia (Bignonaceae) & 95 \\
Palometa caspi & Neea parvifolia (Nyctaginaceae) & 80 \\
Anallo caspi & Cordia alliodora (Boraginaceae) & 84 \\
\hline
\end{tabular}

* Lao (1986), Vásquez (1989), Vásquez \& Gentry (1989), Pinedo-Vásquez \& Chota-Inuma (1990), Phillips et al. (1994)

Patrones similares de infección para las familias de leguminosas y una amplia variación en las moráceas se encontraron en el barbecho de bosque secundario de 16 años. Estos resultados están de acuerdo con los encontrados por St. John (1980b) en una evaluación realizada en un bosque húmedo en un oxisol cerca de Manaus, Brasil.

Él atribuyó sus hallazgos a la relación que existe entre la profundidad de la raíz y el nivel de infección micorrícica, es decir, especies que enraizan superficialmente es más probable que sean infectadas y que tomen fósforo más eficientemente en comparación con especies que enraizan a mayor profundidad. Las diferencias en colonización micorrícica entre las especies evaluadas están probablemente relacionadas a su profundidad de enraizamiento. Adicionalmente, la raíces que proliferan en las capas de hojarasca y humus (Díaz \& Rivera 2001) parecen que soportan mayores niveles de infección que las raíces que crecen en el suelo (Rose \& Paranka 1987).

Es interesante notar que se presenta una ligera tendencia de existir mayores niveles de infección micorrícica en la repetición II que en las repeticiones I y III para los dos barbechos de bosque, donde el contenido de arcilla del suelo es de 19\% (rep. II) y 10 y $8 \%$ (repeticiones I y III) respectivamente. Esta tendencia puede estar relacionada con la disponibilidad de fósforo en el suelo, la que es de 25 a 50\% mayor en el suelo de las repeticiones I y III. (Uribe et al. 1991).

Solamente cuatro especies arbóreas estuvieron presentes en las tres repeticiones del experimento. Inga sp., Heliocarpus popayanensis, Guatteria sp. y Piper sp. (tabla 5). En la estación seca, no se presentaron diferencias significativas en los niveles de infección entre las especies de árboles. El nivel de infección fue alto y no hubo diferencias para Inga sp., Guatteria sp. y Piper sp. H. popayanensis tuvo un nivel de infección ligeramente inferior comparada con las otras especies, aunque estos niveles tuvieron una gran variabilidad entre las tres repeticiones. En la estación húmeda sin embargo, la infección en Inga sp., fue significativamente más alta que en la otras especies $(\mathrm{p}=0.012)$. La mayoría de las leguminosas arbóreas en la Amazonía peruana parece que soportan niveles altos de infección micorrícica (Ruiz et al. 1989).

Tabla5. Colonización micorrícica del barbecho de bosque secundario de cinco años. Estación seca (agosto, 1991) y estación húmeda (diciembre, 1992). Los números entre paréntesis son las desviaciones estándar.

\begin{tabular}{|l|c|c|}
\hline \multirow{2}{*}{ Especie } & \multicolumn{2}{|c|}{ Micorriza (\%) } \\
\cline { 2 - 3 } & Estación seca & Estación húmeda \\
\hline Inga sp. & $84(10.0)$ & $86(13.8)$ \\
Heliocarpus popayanensis & $64(31.6)$ & $45(19.8)$ \\
Guatteria sp. & $87(1.7)$ & $60(4.2)$ \\
Piper sp. & $85(4.5)$ & $48(17.1)$ \\
\hline
\end{tabular}

$\mathrm{n}=3$ 
Se encontró una diferencia significativa $(\mathrm{p}=0.043)$ en los niveles de infección entre las dos estaciones del año. La infección fue más alta en la estación seca, particularmente para H. popayanensis, Guatteria sp. y Piper sp. El nivel alto de infección en Inga sp. se mantuvo durante las dos estaciones. Las diferencias en infección entre las dos estaciones se relacionan probablemente al estado de humedad del suelo. Redhead (1975) demostró que la provisión óptima de agua para el crecimiento de la planta es también adecuada para la infección micorrícica. Parece que un exceso de agua durante la estación lluviosa afectó la actividad de las micorrizas (figura 1). La provisión de agua para las micorrizas son especialmente importantes en condiciones de sequía (Sieverding 1981). Es probable que en esta situación estén involucrados dos mecanismos: el mejoramiento de la absorción de agua por la planta y el mejoramiento de la utilización del agua (Dommergues 1992). Parece entonces, que el régimen de la humedad údico del suelo en la Amazonía del Perú, el que permite periodos cortos de sequedad del suelo (Sánchez, 1976) pueda haber influido por la variabilidad en los niveles de infección mocorrícica del sistema. Periodos de sequedad del suelo, sin embargo, parecen no afectar la infección en algunos árboles, tal es el caso de Inga. sp.

Sistema de producción en multiestratos

No hubo diferencias significativas en colonización micorrícica entre las especies en el sistema en multiestratos (tabla 6). Los valores de colonización fueron altos y comparables a aquellos de los barbechos de bosque. Esta tendencia se encontró también entre las estaciones evaluadas. La variabilidad entre los valores de colonización, sin embargo, fue alta para algunas especies. Los niveles del porcentaje de colonización fueron más consistentes (valores bajos de desviación estándar) para Centrosema macrocarpum y Theobroma cacao (estación seca) y para Cedrelinga catanaeformis e Inga edulis (estación húmeda).

Los niveles relativamente altos de colonización micorrícica encontrados en este sistema están probablemente relacionados a un aumento en la competencia por nutrientes, particularmente fósforo, entre las especies. Alegre et al. (1989), reportaron que se extrajeron hasta $26 \mathrm{~kg} \mathrm{ha}^{-1}$ de $\mathrm{P}$ de este sistema en productos cosechados en cuatro años, comparados con adiciones mucho más pequeñas como insumos atmosféricos. Palm et al. (1991) reportaron que a pesar de las altas tasas de pérdida de $\mathrm{P}$ del sistema y las no adicionales de fertilizante fosfatados, los árboles continuaron creciendo rápidamente a niveles de P por debajo del considerado adecuado para la producción de cultivos. Un mecanismo posible por el cual los árboles pueden mantener tasas altas de crecimiento y producción bajo estas condiciones está probablemente relacionado a una mayor actividad micorrícica en tomar reservas de P, particularmente en las capas de hojarasca (Rose \& Paranka 1987; Díaz 2001). Otro posible mecanismo incluye el uso de formas de $\mathrm{P}$ menos disponibles por los árboles (Palm et al. 1991), o un aumento en ciclo interno del P de los árboles al envejecer (Davey, C.B., comunicación personal).

Tabla 6. Colonización micorrícica en el sistema de producción en multiestratos. Estación seca (agosto, 1991) y estación húmeda (diciembre, 1992). Los números entre paréntesis son las desviaciones estándar.

\begin{tabular}{|l|c|c|}
\hline \multirow{2}{*}{ Especie } & \multicolumn{2}{|c|}{ Micorriza (\%) } \\
\cline { 2 - 3 } & Estación seca & Estación húmeda \\
\hline Centrocema macrocarpum & 78 & 69 \\
& $(5.2)$ & $(16.1)$ \\
Cedrelinga catanaeformis & 56 & 79 \\
& $(35.8)$ & $(4.2)$ \\
Bactris gasipaes & 65 & 59 \\
& $(17.5)$ & $(22.5)$ \\
Collubrina glandulosa & 81 & 57 \\
& & \\
\hline
\end{tabular}




\begin{tabular}{|l|c|c|}
\hline Eugenia stipitata & 78 & 52 \\
Inga edulis & $(19.4)$ & $(11.2)$ \\
& 71 & 91 \\
Theobroma cacao & $(30.3)$ & $(2.5)$ \\
& 88 & 75 \\
\hline
\end{tabular}

$\mathrm{n}=3$

La diferencia entre el sistema de producción en multiestratos y los barbechos de bosque secundario, es que en los últimos no existe una pérdida de P por medio de cosechas (Palm et al. 1991) y los altos niveles de colonización micorrícica en los bosques secundarios se deben probablemente a la competencia resultante de una mayor densidad y diversidad de plantas (Janos 1984) que en el sistema en multiestratos.

La variabilidad de los niveles de colonización en algunas especies de árboles están probablemente relacionadas a las labores de manejo, p.e., podas, siembras, ect., coincidentes con el muestreo de las raíces. Se encontró que en las podas de las ramas resultaron en la mortalidad de las raíces y en la reducción en el porcentaje de la longitud de raíces colonizadas con micorrizas (Fernández 1990). La relativamente pequeña diferencia en los niveles de colonización micorrícica entre las estaciones podría deberse a los efectos de la cobertura leguminosa en el sistema en relación al mantenimiento de condiciones adecuadas de humedad dek suelo (Wade \& Sánchez 1983) para la actividad micorrícica.

Plantación de pijuayo

No hubo diferencias significativas en la colonización micorrícica entre las especies de la plantación de pijuayo (tabla 7). Los niveles de colonización en Bactris gasipaes y Centrosema macrocarpum fueron similares como en el sistema en multiestratos. De manera similar, no se observaron variaciones en la colonización entre las dos estaciones. Se encontró, sin embargo, alguna variabilidad entre las repeticiones.

Tabla 7. Colonización micorrícica en la plantación de pijuayo. Estación seca (agosto, 1991) y estación húmeda (diciembre, 1992). Los números entre paréntesis son las desviaciones estándar.

\begin{tabular}{|l|c|c|}
\hline \multirow{2}{*}{ Especie } & \multicolumn{2}{|c|}{ Micorriza (\%) } \\
\cline { 2 - 3 } & Estación seca & Estación húmeda \\
\hline Bactris gasipaes & 56 & 68 \\
Centrosema macrocarpum & $(14.6)$ & $(14.5)$ \\
& 45 & 62 \\
& $(15.5)$ & $(21.4)$ \\
\hline
\end{tabular}

$\mathrm{n}=3$

Los frutos de pijuayo se cosechan anualmente, representando una pérdida de P la que tiene que ser restituida para mantener la producción de frutos. Tal como se indicó para el sistema en multiestratos, un posible mecanismo de los árboles de pijuayo para tomar P eficientemente es a través de las micorrizas. Arévalo et al. (1993) reportaron que no hubieron cambios significativos en $\mathrm{P}$ durante 5 años después del establecimiento de ambos sistemas (cerca de 9 ppm). Es posible también, que formas de $\mathrm{P}$ menos disponibles estén siendo usadas por el pijuayo, tal como lo sugiere Palm et al. (1991). Tal como se esperaba, la colonización micorrícica en la cobertura leguminosa fue alta. Se sabe que la mayoría de especies leguminosas que nodulan soportan niveles altos de colonización micorrícica para su crecimiento y fijación de nitrógeno, particularmente en suelos ácidos (Barea \& Azcón-Aguilar 1983; Fernandes 1990; Ruiz et al., 1989). 


\section{Cultivos continuos con bajos insumos}

Este sistema se inició con una rotación de arroz-arroz-caupí. Luego le siguió un cultivo más de arroz y luego se sembró kudzú en forma intercalada. Después del barbecho de kudzú de 18 meses, se inició un segundo ciclo de cultivos (1989). Luego se sembró de nuevo kudzú y se aplicó roca fosfata a una dosis de $10 \mathrm{~kg}$ de P / ha. El barbecho de kudzú permaneció hasta septiembre de 1992, cuando se plantó un tercer ciclo de cultivos (Alegre, J.C., comunicación personal).

Las especies evaluadas fueron kudzú (estación seca) y arroz (estación húmeda), respectivamente (tabla 8). La colonización fue más alta en kudzú que en arroz. Esta diferencia puede estar relacionada a la dependencia relativa de los cultivos a las micorrizas para tomar fósforo. Se sabe que la mayoría de las leguminosas tiene una alta demanda de P para su crecimiento, nodulación y fijación de nitrógeno y que a menudo no pueden crecer en suelos ácidos con bajos niveles de P disponible (Barea \& Azcón-Aguilar 1983). La investigación ha mostrado que asociaciones efectivas entre muchas especies leguminosas y hongos MA mejoran significativamente el crecimiento y la fijación de nitrógeno en relación a plantas no micorrícicas (Barea \& Azcón-Aguilar 1983; Schoeneberger et al. 1989). Los bajos niveles de colonización micorrícica en las plantas de arroz están probablemente asociados con las características de sus raíces, las que son muy finas y con abundantes pelos radiculares (Baylis 1975). Niveles similares de colonización micorrícica fueron observados por Ruiz et al. (1989) en un estudio previo conducido en la misma región. Los requerimientos de fósforo para la biomasa de kudzú y la producción de arroz fueron posiblemente conseguidos de la aplicación de P en 1990.

Tabla 8. Colonización micorrícica en el sistema de cultivos continuos con bajos insumos. Estación seca (agosto, 1991) y estación húmeda (diciembre, 1992). Los números entre paréntesis son las desviaciones estándar.

\begin{tabular}{|l|c|c|}
\hline \multirow{2}{*}{ Especie } & \multicolumn{2}{|c|}{ Micorriza (\%) } \\
\cline { 2 - 3 } & Estación seca & Estación húmeda \\
\hline Pueraria phaseoloides & 52 & - \\
Oryza sativa & $(22.1)$ & 13 \\
& - & (4.9) \\
\hline
\end{tabular}

En general, los niveles de colonización micorrícica en el sistema con bajos insumos fueron más bajos que en los bosques secundarios y en el sistema en multiestratos.

Estos bajos niveles posiblemente estén relacionados a los cambios a largo plazo en la composición de las comunidades de plantas y en las propiedades físicas y químicas del suelo que afectaron las micorrizas (Abbott \& Robson, $1991^{\mathrm{a}}$ ).

Es interesante la tendencia en relación a altos niveles de colonización micorrícica en la repetición II (más arcilla/ menos P disponible) que se encontró en algunas especies. En el sistema con bajos insumos (Pueraria phaseoloides); en el sistema en multiestratos (Bactris gasipaes, Theobroma cacao, Centrosema macrocarpum y Cedrelinga catanaeformis); y en la plantación de pijuayo (Bactris gasipaes y C. macrocarpum). Las figuras 3 y 4 muestran esta tendencia para Bactris gasipaes, en el sistema en multiestratos y en la plantación de pijuayo. Para las otras especies incluidas en su respectivo sistema, esta tendencia fue menos clara. Se debe mencionar que en los tres sistemas mencionados, la producción de cultivos estuvo correlacionada positivamente con el porcentaje de arcilla en el suelo superficial (Alegre et al. 1991). 


\section{Cultivos continuos con altos insumos}

Este sistema fue iniciado con una rotación de maíz-soya la que se continuó hasta 1990. En 1991 se inició una rotación de maíz-maní hasta 1992. De setiembre 1985 a diciembre 1992, se realizaron 15 cosechas y el sistema recibió un total de 890, 474, 1393, 4000 y $500 \mathrm{~kg}$ / ha de N, P, K, cal dolomítica y Ca (OH), respectivamente. Adicionalmente, el terreno fue mecanizado y recibió aplicaciones de herbicidas para el control de malezas (Alegre, J.C., comunicación personal).

Las especies evaluadas fueron maíz (Zea mays) en la estación seca y maní (Arachis hypogaea) en la estación húmeda (tabla 9). La colonización micorrícica fue baja para los dos cultivos, pero más baja en maní. El muestreo de raíces se hizo en los estados iniciales de crecimiento para ambos cultivos (15 días después de la siembra). Un estudio de vivero conducido en el mismo lugar mostró niveles altos de colonización micorrícica en plantas de maíz a los 14 días después de la siembra (Ruiz \& Scholes 1989). Los bajos niveles de colonización en estos cultivos están probablemente relacionados a los efectos de la alteración del suelo sobre hongos MA durante la mecanización. Estudios de invernadero mostraron que la ruptura del micelio extra-radical por la alteración del suelo reduce la colonización micorrícica en los cultivos (Jasper et al. 1989; Evans \& Millar 1990; Millar \& McGonigle 1992). Los propágulos del hongo MA posiblemente se mantengan viables y retengan su efectividad de un cultivo a otro, si el suelo no es alterado por la mecanización. Otro efecto de la alteración del suelo en la formación de la micorriza puede estar asociado con la reducida absorción de fosfato después de la mecanización comparada con la absorción sin mecanización (O’Halloran et al. 1986). Estos efectos, sin embargo, parecen diferir con las especies de plantas (Evans \& Millar 1988).

Tabla 9. Colonización micorrícica en el sistema de cultivos continuos con altos insumos. Estación seca (agosto 1991) y estación húmeda (diciembre, 1992). Los números entre paréntesis son las desviaciones estándar.

\begin{tabular}{|l|c|c|}
\hline \multirow{2}{*}{ Especie } & \multicolumn{2}{|c|}{ Micorriza (\%) } \\
\cline { 2 - 3 } & Estación seca & Estación húmeda \\
\hline Zea mays & 47 & - \\
Arachis hypogaea & $(17.5)$ & 16 \\
& - & $(7.1)$ \\
\hline
\end{tabular}

$\mathrm{n}=3$

Los altos niveles de P en el suelo podrían también dar cuenta por los bajos niveles de colonización micorrícica en el maíz y maní (Hayman et al. 1975; Kucey \& Paul 1983). En un estudio de invernadero conducido por la Amazonía del Perú, Ruiz y Scholes (1989) encontraron los niveles más bajos de colonización micorrícica en plantas de maíz que crecieron en un sistema con altos insumos por 14 años. El maní, como la mayoría de las leguminosas, se beneficia de las micorrizas (Krishna \& Bajyaraj 1982), su respuesta a la colonización micorrícica, sin embargo, puede ser dependiente del fenotipo (Krishna et al. 1984; Kesava Rao et al. 1990). Las adiciones de fertilizantes fosfatos podría haber aumentado la longitud de las raíces al grado de disminuir el porcentaje de colonización (Abbott \& Robson 1977).

Cambios en la acidez del suelo, mediante el encalado, pueden adicionalmente afectar la habilidad de las especies de hongos MA para colonizar y persistir en el suelo. Estos pueden incluir efectos en la supervivencia y germinación de las esporas, en el crecimiento de la hifas en el suelo, en la penetración y colonización de la raíz y en la formación del propágulo (Robson \& Abbott 1989). 
La presencia de especies que no forman micorrizas podrían también haber reducido la formación de la micorriza en los cultivos al producir compuestos tóxicos que afectan a los hongos MA (Hayman et al. 1975; Abbott \& Robson 1990). Se observó la proliferación de especies que no forman micorrizas (Cyperus spp., Commelina difusa y Portulaca oleracelas) en las parcelas con altos insumos evaluadas, así como también en otras parcelas fertilizadas en la Estación Experimental de Yurimaguas (Mt. Pleasant 1987).

\section{Características morfológicas de los hongos MA en las raíces}

En la tabla 10 se presenta una clave para la identificación de las características morfológicas de los hongos MA. Las tablas 11, 12 y 13 ilustran la gran diversidad de los patrones de colonización encontrados en las raíces en los barbechos de bosque secundario estudiados.

La presencia de esporas con sus hifas sostenedoras adheridas a las raíces, o el patrón de desarrollo de las hifas en las raíces, o las células auxiliares, sólo en los géneros Gigaspora y Scutellospora, podría identificar asociaciones preferenciales entre los hongos MA y especies de plantas hospederas. Tal como indican Abbott \& Gazey (1994), las asociaciones preferenciales en el campo necesitan ser corroboradas mediante estudios más controlados.

Tabla 10. Clave para la identificación de algunas estructuras de hongos de micorriza arbuscular en las raíces de las especies de plantas en los tratamientos en estudio.

\begin{tabular}{ll}
\hline V & Vesículas \\
A & Arbúsculos \\
HG & Hifas gruasas \\
HF & Hifas finas \\
HMF & Hifas muy finas \\
CAE & Células auxiliares equinuladas \\
CA & Células auxiliares \\
Ep & Esporocarpos \\
E & Esporas \\
Re & Racimo de esporas de Glomus spp. \\
HP & Hifas tipo Paris \\
HA & Hifas tipo Arum \\
PRc & Pelos radiculares cortos \\
Ab & Abundante \\
\hline
\end{tabular}

Tabla 11. Algunas características morfológicas de hongos formadores de micorriza arbuscular en las raíces de las especies en el bosque secundario de 16 años.

\begin{tabular}{ll}
\hline Especie & Características del hongo \\
\hline Inga sp. & Ab HF, HP. CAE \\
Ciprana & PRc, HF, HG, HA, E (Glomus) \\
Belklucia grossularoides & HF, HG, A \\
Piper sp. & HF, V \\
Vismia sp. & HG, HF, E, Re (Glomus), CAE, A \\
Ficus anthelmintica & HG, HF, V, A \\
Pourouma cecropiifolia & HG, HF, V \\
Siparuna sp. & HG, HF, V
\end{tabular}


Cecropia sp.

Aniba sp.

Iryanthera $\mathrm{sp}$

Jacaranda copaia

Miconia petiolaris

Cabralea canjerana

Isertia sp.

Cynometra sp.

Cedrela

Yacocaspi

Ceiba sp.

Sloanea aff. Megaphylla

Trema micrantha

Chucchuvara

Guatteria sp.

Compsoneura capitelata

Diplotropis martiusii

Hornopichana

Unonopsis floribunda

Virola flexuosa

Terminalia sp.

Cosmibuena grandiflora

Lonchocarpus sp.
PRI, HMF, HG, HA, V, HP

HG, HF, HA, E (Glomus)

$\mathrm{HF}$

HMF, HF, HA, V, A, CAE, E (Gigaspora)

$\mathrm{HF}$

HF, HG, V, E (Scutellospora, Glomus)

HMF

PRc, Ab PRI, Ab HG

HG CA

Ab PRI, HG

HF, V

PRI, HF, HG, A, V, E (Glomus)

HG, V,E

$\mathrm{HF}$

HMF, HF, E

PRc, HC, HP

HGV

HG

HF, HG, HA, V

HG

HG

Ab PR, HG

HF, HG

Tabla 12. Algunas características morfológicas de hongos formadores de micorriza arbuscular en las raíces de las especies en el bosque secundario de cinco años.

\section{Especie}

Guatteria sp.

Ambelania occidentalis

Piper sp.

Inga sp.

Unonopsis floribunda

Azarsisa

Cecropia sp.

Lonchocarpus utilis

Theobroma sp.

Ubiamba

Alchornea sp.

Trema micrantha

Heliocarpus popayanensis

Ficus anthelmintica

Cedrelinga catanaeformis

Cynometra sp.

Clycophyllum sprucaenum

Vismia tomentosa

\section{Características del hongo}

Ab HG, HF, V, A, HP

HG HP

Ab PR, HF, HG, V, E (Glomus)

HG, Ep, V, E (Glomus)

Ab HF, HG, V

PRc, Ab HG, HF, A, CAE

HG, HF

$\mathrm{HG}$

HF, HG

HG V, HP

HF, HG, V

HG CAE

HF, HG, HA, V

HG, HF, V

$\mathrm{HG}$

HG, HF, V, E (Glomus)

HG, A

HG 


$\begin{array}{ll}\text { Neea parvifolia } & \mathrm{HG}, \mathrm{HF}, \mathrm{E} \text { (Glomus) } \\ \text { Apeiba sp. } & \mathrm{HG} \\ \text { Pourouma laevis } & \mathrm{PRc}, \mathrm{Hg}, \mathrm{CAE} \\ \text { Tamborhuayo } & \mathrm{HF} \\ \text { Casearia arborea } & \mathrm{HF} \\ \text { Cabralea canjerana } & \mathrm{Ab} \text { PRI, HG } \\ \text { Jacaranda copaia } & \mathrm{V}, \mathrm{E} \text { (Glomus) } \\ \text { Pourouma caussopoa } & \mathrm{HF}, \mathrm{V} \\ \text { Siparuna sp. } & \mathrm{HF} \\ \text { Ochroma lagopus } & \mathrm{HG}, \mathrm{HF} \\ \text { Solanum hazenii } & \mathrm{HF}\end{array}$

Se debe señalar que los hongos micorrícicos arbusculares al orden Glomales, subórdenes Glomineae y Gigasporineae, familias Glomaceae (género Glomus), Acaulosporaceae (géneros Entrophospora y Acaulospora), Archaeosporaceae (género Archaeospora), Paraglomaceae (género Paraglomus) y Gigasporaceae (géneros Gigaspora y Scutellospora) (Morton \& Benny 1990; Morton \& Redecker 2000).

En algunas especies de árboles, se encontraron solamente hifas, ya sea gruesas (HG), finas (HF) o muy finas (HMF). En este estado es imposible identificar asociaciones preferenciales entre los hongos MA y plantas hospederas, ya que todos los hongos MA forman hifas. Similarmente, los arbúsculos (A) son formados por todos los géneros. Las vesículas (V) sólo las forman los géneros Glomus, Acaulospora, Entrophospora, Paraglomus y Archaeospora.

En el barbecho de bosque secundario de 16 años, las especies con pelos radiculares largos (RL) como Cecropia sp. Cosmibuena grandiflora, Cynometra, yacocaspi, Sloanea aff. megaphylla, alcanzaron niveles más bajos de colonización micorrícica comparados con especies sin pelos radiculares (Tabla 2). Se encontraron esporas de Glomus sp. En la corteza de algunas raíces, mientras que esporas de Scutellospora y Gigaspora fueron observadas adheridas a las raíces de dos especies arbóreas.

En el bosque secundario de cinco años, se observó la presencia de pelos radiculares largos y niveles de colonización micorrícica bajos en la especie Cabralea canjerana (tablas 12 y 4, respectivamente). Se encontraron solamente esporas de Glomus sp. en la corteza de algunos de los árboles.

Aunque la abundancia de cualquiera de las estructuras morfológicas de los hongos MA no fueron cuantificados (por ejemplo, número $\mathrm{cm}^{-1}$ de raíz), algunas especies mostraron claramente abundantes hifas (AB) o vesículas en sus raíces. La presencia de arbúsculos fue observada raramente, debido tal vez a su corto tiempo de vida (7-14 días) después que las ramificaciones arbusculares se deterioran y colapsan (Bonfante-Fasolo 1984). Otras estructuras como hifas tipo Paris (HP), esporocarpos (Ep), racimos de esporas (Re), se observaron raramente. Esto se debe probablemente al hecho que estas estructuras son formadas por pocas especies de hongos MA.

En la mayoría de especies en el sistema de multiestratos, se observaron hifas gruesas. Se encontraron esporas de Glomus sp. en la corteza radicular de Inga edulis, sugiriendo alguna afinidad. En la plantación de pijuayo se encontraron también esporas de Glomus sp. en la corteza de Bactris gasipaes. En el sistema de bajos insumos se encontraron adheridas a las raíces de Pueraria phaseolodes esporas de Scutellospora sp. y en el sistema de altos insumos, esporas de Gigaspora sp. adheridas a las raíces de Zea mays, sugiriendo asimismo, relaciones de afinidad entre las especies de hongos micorrícicos y las plantas referidas. 


\section{Especies de hongos de micorriza arbuscular}

Especies del género Glomus estuvieron presentes en los seis sistemas de manejo estudiados, siendo más abundantes en el bosque secundario de cinco años, en la plantación de pijuayo y en el sistema de bajos insumos (tabla 14). Especies del género Acaulospora predominaron en el bosque secundario de 16 años y en el sistema en multiestratos, aunque estuvieron presentes en los otros sistemas, excepto en el sistema con altos insumos. Janos \& Trappe (1982) reportaron que Acaulospora sp. predomina en los suelos ácidos tropicales, entonces, su ausencia en el sistema de altos insumos pudo ser debido a los efectos de las aplicaciones de cal disminuyendo la acidez del suelo.

Tabla 14. Diversidad de especies de hongos de micorriza arbuscular en Ultisoles bajo diferentes opciones de manejo.

\begin{tabular}{|c|c|c|c|c|}
\hline Manejo del suelo & Gomus & $\begin{array}{l}\text { Acaulospora } \\
\text { Número de esp }\end{array}$ & $\begin{array}{l}\text { Gigaspora } \\
100 \text { g suelo seco }\end{array}$ & Scutellospora \\
\hline $\begin{array}{l}\text { Bosque secundario } \\
\text { de } 16 \text { años }\end{array}$ & 21 & 73 & 1 & 0 \\
\hline $\begin{array}{l}\text { Bosque secundario } \\
\text { de } 5 \text { años }\end{array}$ & 88 & 22 & 0 & 0 \\
\hline $\begin{array}{l}\text { Sistema en } \\
\text { multiestratos }\end{array}$ & 39 & 58 & 2 & 0 \\
\hline $\begin{array}{l}\text { Plantación de } \\
\text { pijuayo }\end{array}$ & 344 & 202 & 6 & 1 \\
\hline $\begin{array}{l}\text { Sistema de } \\
\text { cultivos continuos } \\
\text { con bajos insumos }\end{array}$ & 52 & 4 & 0 & 134 \\
\hline $\begin{array}{l}\text { Sistema de } \\
\text { cultivos continuos } \\
\text { con altos insumos }\end{array}$ & 93 & 0 & 14 & 0 \\
\hline
\end{tabular}

Las esporas de los hongos MA no están presentes de manera uniforme en los suelos y su recuperación en un área determinada puede requerir un muestreo extensivo. Debido a limitaciones de tiempo en el área de estudio, se analizó sólo una muestra compuesta de las tres repeticiones de cada sistema. La alta variabilidad en cuanto al número de esporas encontrada en este estudio se debió probablemente al método de muestreo utilizado.

Esporas del género Scutellospora parecen estar asociadas preferencialmente con la leguminosa Pueraria phaseoloides en el sistema de bajos insumos. Se encontraron pocas esporas de Gigaspora sp. en todos los sistemas de estudios.

La predominancia relativa de cualquiera de las especies de hongos micorrícicos en los sistemas evaluados, parece estar afectado por el manejo del suelo. Esto indica cambios en las propiedades físicas, químicas y probablemente biológicas. El cambio en la composición florística puede haber afectado también la diversidad de las poblaciones de los hongos MA. 


\section{CONCLUSIONES}

La colonización de hongos de micorrizas arbusculares es alta en la mayoría de árboles en barbechos de bosque secundario en la Amazonía del Perú. Algunas especies de árboles con pelos radiculares largos alcanzaron niveles más bajos de colonización que especies sin estas estructuras. El grado de colonización micorrícica en algunas especies es alto en la estación seca, mientras que en otras es alto es la estación húmeda. En algunos árboles, la colonización micorrícica parece aumentar con el contenido de arcilla en el suelo. Los cultivos anuales alcanzaron los niveles más bajos de colonización.

Los patrones de colonización micorrícica fueron muy variables entre la especies de plantas evaluadas. Se podría establecer asociaciones preferenciales entre especies de hongos MA y plantas detectando algunas estructuras morfológicas inherentes a una determinada especie de hongo MA en las raíces de las plantas. Estas asociaciones, sin embargo, necesitan ser corroboradas en condiciones controladas ya sea en el laboratorio o en el invernadero. Las poblaciones de hongos MA parecen estar afectadas por el manejo del suelo. Las especies de Glomus parecen tolerar rangos más amplios de acidez del suelo que Acaulospora sp., que está más restringida en suelos ácidos. Especies de los géneros Scutellospora y Gigaspora fueron las menos predominantes entre las poblaciones de hongos MA en los Ultisoles de la Amazonía peruana. El establecimiento de asociaciones preferenciales entre los hongos MA y especies de plantas puede ser muy valioso para el manejo de especies de árboles, particularmente en el vivero para programas de reforestación o revegetación.

\section{BIBLIOGRAFÍA}

ABBOTT, L.K. AND A.D. ROBSON. 1991a. Factors influencing the occurrence of vesicular-arbuscular mycorrhizas. Agric. Ecosyst. Environ. 35:121-150.

ALEGRE, J.C., J. M. PÉREZ, AND L.T. SZOTT. 1991. Comparative Soil Dynamics: Crop production and soil physical properties. In: T. McBride (Ed.). Trop Soils Annual Report 1988-1989. North Carolina State University, Raleigh. pp. 188-191.

BAYLIS, G.T.S. 1975. The magnolioid mycorrhizae and mycotrophy in root systems derived from it. In: Endomycorrhizas. F.E. Sanders, B. Mosse, and P.B. Tinker (Eds.). Academic Press, London. pp. 373-390.

BONFANTE-FASOLO, P. 1984. Anatomy and morphology of VA mycorrhizae. In: C.LI. Powell and D.J. Bagyraj (Eds.). VA Mycorrhizae. CRC Press. Boca Raton, Florida. pp. 5-33.

CRUSH, J.R. 1976. Endomycorrhizas and legume growth in some soils of the Mackenzie Basin, Canterbury, New Zealand. N.Z. Journal of Agric. Res. 19:473-476.

DÍAZ, L.A. Y RIVERA, E.L. 2001. Hongos de micorriza arbuscular entre hojarasca en proceso de descomposición en ecosistemas naturales y cultivados. Suelos Ecuatoriales Vol. 31, n. ${ }^{\circ}$ 2, 224-249.

DOMMERGUES, I.R. 1992. Management of soil microbial populations to optimize tree establishment and growth, with particular reference to nitrogen-fixing bacteria. In: K. Mulongoy, M. Gueye and D.S.C Spencer (Eds.). Biological Nitrogen Fixation and Sustainability of Tropical Agriculture. Wiley-Sayce CoPublication. pp. 113-131.

FITTER, A.H. 1989. The role and ecological significance of vesicular-arbuscular mycorrhizas in temperate ecosystems. Agric. Ecosyst. Environ. 29:137-151.

GERDEMANN, J.W. 1968. Vesicular-arbuscular mycorrhiza and plant growth. Ann. Rev. Phytopath. 6:397-418. 
GERDEMANN, J.W. AND T.H. NICOLSON. 1963. Spores of mycorrhizal Endogone species extracted from soil by wet sieving and decanting. Trans. Br. Mycol. Soc. 46:235-244.

GIOVANETTI, M. AND B. MOSSE. 1980. An evaluation of techniques for measuring vesicular-arbuscular mycorrhizal infection in roots. New Phytol. 97:447-453.

JANOS, D.P. 1980. Vesicular-arbuscular mycorrhizae affect lowland tropical rain forest plant growth. Ecology 61:151-162.

JANOS, D.P. 1987. VA mycorrhizas in humid tropical acosystems. In: G.R Safir (Ad.). Ecophysiology of VA mycorrhizal plants. CRC Press. Boca Raton, Florida. pp. 107-134.

LAO, R. 1986. Descripción dendrológica de 23 familias de especies forestales del asentamiento rural forestal von Humboldt. Huánuco, Perú. Instituto Nacional de Desarrollo. Proyecto Especial Pichis-Palcazú. LimaPerú. 23 p.

LOPES, E.S. AND J.O. SIQUEIRA. 1980. Vesicular-arbuscular mycorrhizas: their potential in phosphate nutrition in tropical regions. In: R.S. Russell, K. Igue, and Y.R. Metha (Eds.). The Soil/Root System in Relation to Brazilian Agriculture. Proceedings of the Symposium on the Soil/Root System. Instituto Agronómico do Paraná-IAPAR. Londrina, Brazil. pp. 225-242.

MORTON, J.B. AND S.L BEnny. 1990. Revised classification of mycorrhizal fungi (Zygomycetes): a new order, Glomales, two new suborders, Glominae and Gigasporinae, and two new families, Acaulosporaceaeand Gigasporaceae, with a emendation of Glomaceae. Mycotaxon 37:471-491.

MORTON, J.B. AND D. REDECKER. 2000. Concordant morphological and molecular characters reclassify five arbuscular mycorrhizal fungal species into new genera, Archaeospora and Paraglomus, of new families Archaeosporaceae and Paraglomaceae, respectively. Mycologia (in press).

MOSSE, B. 1973. Plant growth responses to vesicular-arbuscular mycorrhiza. IV. In given soil additional phosphate. Ecol. 5:137-210.

NICOLSON, T.H. AND N.C. SCHENCK. 1979. Endogonaceous mycorrhizal endophytes in Florida. Mycologia 71:178-198.

PHILLIPS, O., A.H. GENTRY, C. REYNEL, P. WILKIN, AND C. GALVEZ-DURAND B. 1994. Quantitative ethnobotany and Amazonian conservation. Conservation Biology 8:405-416.

PHILLIPS, J.M. AND D.S. HAYMAN. 1970. Improved procedures for clearing roots and staining parasitic and vesicular-arbuscular mycorrhizal fungi for rapid assessment of infection. Trans. Brit. Mycol. Soc. 55:158161.

PINEDO-VÁSQUEZ, M., D. ZARIN, P. JIPP, AND CHOTA-INUMA, J. 1990. Use-values of tree species in a communal forest reserve in northeast Peru. Conversation Biology 4:405-416.

PUPPI, G. AND S. REISS. 1987. Role and ecology of VA micorrhizae in sand dunes. Angew. Botanik, 61:115-126.

READ, C.P.P. 1990 Mycorrhizas. In: J.M. Lynch (Ed.). The Rhizosphere. Wiley Series in Ecological and Applied Microbiology. John Wiley and Sons. pp. 281-315.

REDHEAD, J.F. 1975. Endotrophic mycorrhizas in Nigeria: some aspects of the ecology of the Endotrophic mycorrhizal associations of Khaya grandiflora C. DC. In: F.E. Sanders, B. Mosse, and P.B. Tinker (Eds.). Endomycorrhizas. Academic Press. pp. 447-468. 
ROSE, S.L. 1988. Above and below ground community development in a marine sand dune ecosystem. Pl. Soil 109:215-266.

RUIZ, P.O. AND M.C. SCHOLES. 1989. Effect of different management options on mycorrhizal infection. In: N.C. Caudle (Ed.). TropSoils Annual Report 1986-1987. North Carolina State University, Raleigh. pp. 116-118.

RUIZ, P.O., M.C. SCHOLES, AND T.V. ST. JOHN. 1989. Occurrence of mycorrhizae in crops, pastures and tree species. In: TropSoils Annual Report 1986-1987. N.C. Caudle (Ed.). North Carolina State University, Raleigh. pp. 115.

SALINAS, J.G. AND P.A. SANCHEZ. 1976. Soil-plant relationships affecting varietal and species differences in tolerance to low available soil phosphorus. Ciencia e Cultura (Brazil) 28:156-168.

SANCHEZ. P.A. 1976. Properties and management of soils in the tropics. John Wiley and Sons. 618 p.

SIEVERDING, E. 1981. Influence of soil water regimes on VAmycorrhiza. I. Effect on plant growth, water utilization, and development of mycorrhiza. Z. Acker-u. Pflanzenbau 150:400-441.

STATISTICAL ANALYSIS SYSTEM. 1985. SAS User’s Guide. SAS Institute, Cary, NC, USA.

ST. JOHN, T.V. 1980a. Una lista de especies de plantas tropicais brasileiras naturalmente infectadas con micorriza vesicular-arbuscular. Acta Amazónica 10:229-234.

ST. JOHN, T.V. 1980b. A survey of micorrhizal infection in Amazonian rain forest. Acta Amazónica 10:527-533.

ST. JOHN. 1980c. Root size, root hairs, and mycorrhizal infection: a re-examination of Baylis's hypothesis with tropical trees. New Phytol. 84:483-487.

ST. JOHN, T.V. AND H.W. HUNT. 1983. Statistical treatment of VAM infection data. PI. Soil 73:307-313.

TSBF (Tropical Soil Biology and Fertility). 1993. A Handbook of Methods. Second Edition. J.M. Anderson and J.S.I. Ingram (Eds.). CAB International. U.K. 221 p.

URIBE, E., L.T. SZOTT, AND J.C. ALEGRE. 1991. Soil fertility dynamics. In: T. McBride (Ed.). TropSoils Annual Report 1988-1989. North Carolina State University. Raleigh, North Carolina. pp. 197-205.

VASQUEZ, R. 1989. Plantas útiles de la Amazonía peruana I. Missouri Botanical Garden. Proyecto Flora del Perú. Iquitos, Perú. 195 p.

VASQUEZ, R. AND A.H. GENTRY. 1989. Use and misuse of forest-harvested fruits in the Iquitos area. Conservation Biology 3:350-361.

WHITTINGHAM, J. AND D.J. READ. 1982. Vesicular-arbuscular micorrhizae in natural vegetation systems. III. Nutrient transfer between plants with mycorrhizal interconnections. New. Phytol. 90:277-284.

WILSON, E.O. 1989. Threats to biodiversity. National Academy Press. Washington, DC. pp. 3-18. 


\title{
PRESENCIA DE MERCURIO EN EL AGUA Y SEDIMENTO DE FONDO EN EL RÍO NANAY, PERÚ
}

\author{
PRESENCE OF MERCURY IN THE BOTTOM SEDIMENTS AND WATERS \\ IN THE NANAY RIVER, PERU
}

\author{
José Maco García ${ }^{1}$ y Elvis Sandoval Zamora²
}

\begin{abstract}
RESUMEN
Evaluaciones de mercurio en el agua y sedimentos de fondo del río Nanay, afluente izquierdo del río Amazonas, ha determinado un claro incremento de las concentraciones promedio que oscilan entre $<20 \mathrm{ng} / \mathrm{l}$ en el año 2000 a 120 ng/l en el 2001. Se identifican lugares con concentraciones superiores a los límites máximos permisibles para aguas de zonas de pesca de mariscos bivalvos (Tipo V) y aguas de zonas de preservación de fauna acuática y pesca recreativa o comercial (Tipo VI) regulados en el Perú. Los sedimentos de fondo presentan concentraciones entre 0.005 a 0.0815 ìg/g, valores considerados por debajo de los límites máximos permisibles establecidos por la EPA(Environmental Protection Agency). En general las fuentes de mercurio del agua provienen parcialmente de los sedimentos del fondo del río debido a su remoción durante la extracción del oro aluvial.
\end{abstract}

Palabras clave: contaminación, mercurio, río Nanay, agua, sedimentos, Amazonía, Perú.

\begin{abstract}
Measurements of mercury in the water and sediments of Nanay river bottom, left affluent of the Amazon river, has determined a clear average increasing of concentrations that range between $<20 \mathrm{ng} / \mathrm{l}$ in the year 2000 to $120 \mathrm{ng} /$ $l$ in the year 2001. There were identified places with concentrations excceding the maximum permissable limits for waters of zones for bivalve seafood fishing (Type V) and waters zones for preservation of aquatical fauna and recreative or commercial fishing (Type VI) regulated by Peruvian law. The sediments of river bottom have concentrations between 0.005 to $0.0815 \mu \mathrm{g} / \mathrm{g}$, values considered below the maximum permissable limits established by the EPA (Environmental Protection Agency). In general, the mercury sources of the water partially become from the sediments of the river bottom due to their removal during the extraction of the alluvial gold.
\end{abstract}

Key words: pollution, mercury, Nanay river, water, sediments, Amazonia, Peru.

\section{INTRODUCCIÓN}

El mercurio, metal muy tóxico para el ambiente y la salud de las poblaciones humanas, es objeto de estudio en varios lugares de la cuenca del río Amazonas (Veiga 1997a; 1997b; Lodenius y Malm 1998; Maurice-Bourgoin et al. 1999; Roulet et al. 1999; 2000a; 2000b, 2001a; 2001b; Carmouze et al. 2001).

En la Amazonía peruana se han realizado muy pocos estudios sobre los niveles de contaminación del mercurio. Algunos estudios se realizaron en la cuenca del río Madre de Dios, donde se sabe que existe una importante actividad minera aurífera en las zonas aluviales que causa serios daños al ambiente y a la salud de la población. Hasta 1994 participaron 3,000 personas en la actividad aurífera en Madre de Dios (GRADE 1994), mientras que en 1999 ese número se elevó a 10,000 personas que trabajan directamente en la minería, en las pequeñas cuencas de Huaypetuhe y Caychive en la cuenca del Madre de Dios (Lundberg 1999). Según IMA (1995) se empleó más de $90 \mathrm{t}$ de mercurio en ocho años de actividad. Hasta 1996, el contenido de mercurio en los cuerpos de agua de la cuenca del Madre de Dios fue de 0.6 a $1.5 \mathrm{ng} / \mathrm{L}$, mientras que los niveles de mercurio en los sedimentos de la cuenca, son del orden de 0.156 a 0.358 ?g/g (Deza 1996).

1 Investigador del Instituto de Investigaciones de la Amazonía Peruana Av. Abelardo Quiñones km 2.5. Apartado 784. Iquitos

- Perú. Correo electrónico: jmaco@iiap.org.pe

2 Funcionario de la Dirección Ejecutiva de Salud Ambiental de Loreto. 
La cuenca del río Nanay es una de las principales fuentes de abastecimiento de alimentos y de aprovisionamiento de agua, la misma que luego de ser potabilizada es distribuida a la ciudad de Iquitos, que alberga una población cercana al de medio millón de habitantes. Esta cuenca es soporte de las actividades socioeconómicas de aproximadamente 25,200 habitantes rurales que se ubican en las orillas del mismo río y sus principales afluentes. En este marco, toda perturbación ambiental en ese importante espacio geográfico, de 1’750,737 ha (IIAP 2002), tiene serias implicaciones regionales, por lo que el desarrollo de las actividades humanas en esta cuenca deben realizarse de forma sostenible teniendo los cuidados necesarios para minimizar la degradación ambiental. En 1994 se realizaron determinaciones de mercurio en el río Nanay, dando como resultado la ausencia de este metal en el agua (Gómez 1994). En esa oportunidad el mercurio no fue detectado, posiblemente debido a las limitaciones referentes a la precisión del método empleado.

A partir de 1999, se reporta el desarrollo de la actividad minera en la cuenca del río Nanay donde se identifican una serie de impactos negativos para el medio ambiente y la salud humana durante el proceso de extracción de minerales (Reyes 2000). Uno de los impactos más severos que la actividad aurífera ocasiona está relacionado con el uso de mercurio durante el proceso de la extracción de oro; el mercurio es un metal sumamente tóxico (Español 2001; Goldman et al. 2001; Clarkson 2002; Kales \& Goldman 2002 y otros). Conocedores de esta problemática, se conformó en la región Loreto una Comisión Técnica Multiinstitucional con la finalidad de evaluar los impactos ocasionados por la extracción de oro en la cuenca del río Nanay. El presente documento incluye parte de los análisis de los estudios realizados por dicha Comisión durante los años 2000 y 2001. Aquí se exponen los resultados de los análisis de mercurio en agua y sedimento de la cuenca del río Nanay.

El río Nanay es el eje hídrico principal de la cuenca del Nanay que está localizada en el llano amazónico, en la parte noreste del territorio del Perú. Pertenece a la provincia de Maynas, región Loreto, y geográficamente se encuentra ubicada entre los meridianos $73^{\circ} 13^{\prime}$ y $75^{\circ} 03^{\prime}$ longitud oeste y los paralelos $2^{\circ} 33^{\prime}$ y $4^{\circ} 02^{\prime}$ latitud sur (figura 1 ).

El río Nanay nace en el llano amazónico y desemboca en la margen izquierda del río Amazonas a la altura de la ciudad de Iquitos. Es un río pequeño cuyo caudal oscila entre 70 a 200 m³/s (AUDITEC SAC-DINAMICA 2000). Presenta características limnológicas que corresponden a aguas negras como las descritas por Sioli (1984), las mismas que se resumen en tener moderada transparencia, alrededor de un metro de profundidad de la columna de agua, oxígeno disuelto de 2.5 a 4.7 mg/L, un pH ligeramente ácido de 6.3 a 6.6 (Gómez 1994) y coloración negruzca; su caudal es de alrededor de 240 m³/s (IIAP 1999a; 1999b; 2002).

En el ámbito de su cuenca se realizan una serie de actividades económicas, destacando las siguientes: la extracción de madera y de productos diferentes a la madera, agricultura de subsistencia, caza de subsistencia, pesca de subsistencia y comercial, así como la extracción oro aluvial y material de acarreo. La extracción de estos minerales comenzó en 1999 (Reyes 2000) empleándose para el efecto «dragas de succión», que remueven el fondo del río hasta una profundidad de un metro. De acuerdo a los estudios de impacto ambiental, las dragas tienen una capacidad de succión de $60 \mathrm{~m}^{3} /$ hora de sedimentos y trabajan un promedio de 15 horas por día (AUDITEC SACDINAMICA 2000). Sin embargo, los pobladores del río Nanay mencionan que las dragas trabajaban las 24 horas del día. En el río Nanay, la ley del oro es de alrededor de $0.3 \mathrm{~g} / \mathrm{m}^{3}$. y durante el proceso de amalgamado se usa mercurio metálico, en cantidades que oscilan entre dos a tres veces la cantidad de oro encontrado. 


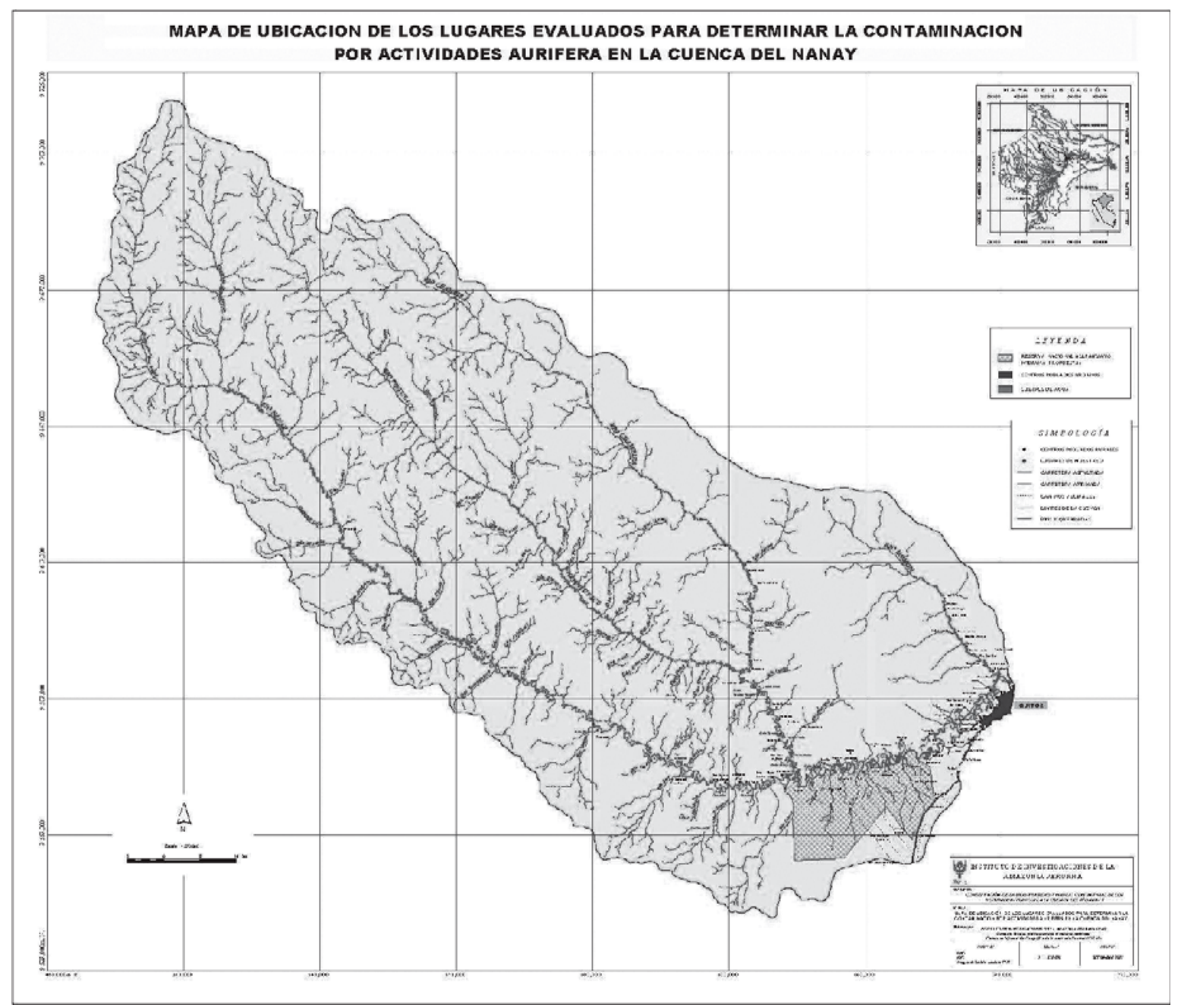

Figura 1.

\section{MATERIAL Y MÉTODO}

La toma de muestras del río Nanay se realizó durante mayo de 2000, y mayo y octubre de 2001. En el 2002, se establecieron cuatro estaciones de muestreo donde se colectó una muestra de agua del centro del río, por estación. Durante el año 2001 se establecieron 15 estaciones de muestreo y se tomó una muestra de agua del centro del río por estación. Las muestras de agua fueron colectadas en botellas de teflón de un litro de capacidad y fueron adecuadamente preservadas con $1 \mathrm{ml}$ de ácido nítrico concentrado y refrigeradas a $4^{\circ} \mathrm{C}$ hasta su envío al laboratorio.

Solamente en octubre de 2001, paralelamente a la toma de muestras de agua, se colectaron muestras de sedimentos del lecho del río en las 15 estaciones de muestreo. Se empleó una draga tipo Eckman y las muestras fueron colocadas en bolsas de polietileno, etiquetadas y refrigeradas a $4^{\circ} \mathrm{C}$ hasta su envío al laboratorio.

Posteriormente, las muestras de agua y sedimentos colectados fueron remitidos a la ciudad de Lima para ser analizados en los laboratorios de la Dirección General de Salud Ambiental-DIGESA del Ministerio de Salud del Perú. El análisis de mercurio se realizó a través del método Oficial Method AOAC 986.15 Multielement Methods: arrastre por vapor frío sin flama - absorción atómica. 


\section{RESULTADOS}

\section{Mercurio en agua}

La concentración de mercurio total medido en las aguas superficiales del río Nanay se incrementa a través del tiempo. En los muestreos realizados durante el año 2000, las concentraciones de mercurio total fueron menores de 20 ng/L. Estos resultados son inferiores a las concentraciones de los límites máximos permisibles establecidos por el Perú para los diferentes tipos de agua de acuerdo a sus usos (El Peruano 1983). Esos límites oscilan entre 100 ng/L y 2,000 ng/L de mercurio. Sin embargo, en el 2001 las concentraciones de mercurio en la cuenca del río Nanay se incrementaron (figuras 2 y 3), llegando en algunos casos a sobrepasar los valores de los límites máximos permisibles para los tipos de agua V (aguas de zonas de pesca de mariscos bivalvos) y VI (aguas de zonas de preservación de fauna acuática y pesca recreativa o comercial).

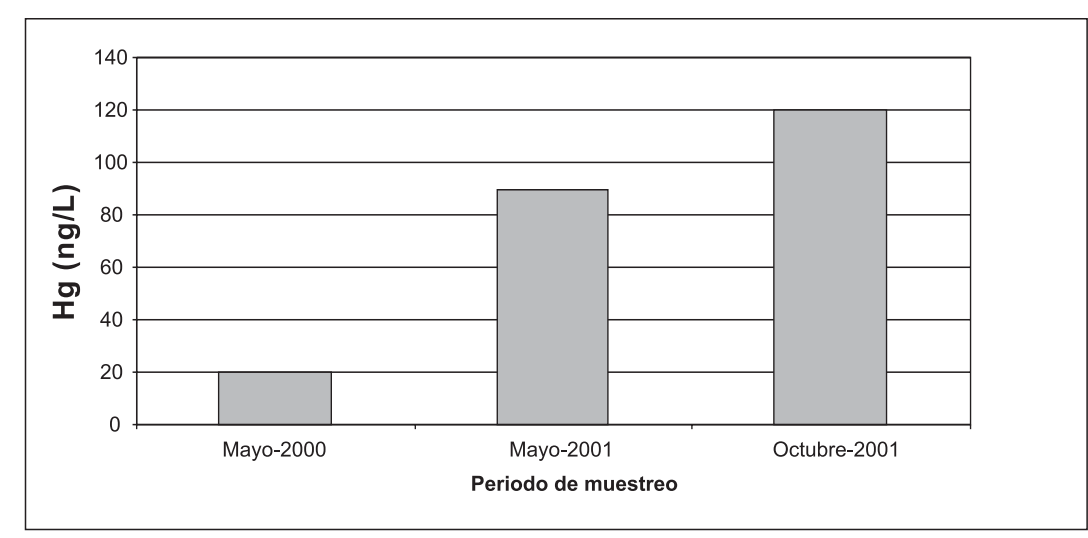

\section{Nótese el incremento de mercurio del año 2000 al 2001 en el río Nanay.}

Figura 2. Contenido promedio de mercurio en agua (mg/L) del río Nanay. Fuente: DIGESA

En el mes de mayo del 2001, que corresponde al periodo de creciente del río, la concentración de mercurio en el río Nanay varió entre <20 a 920 ng/L, siendo el promedio de 90 ng/L (n=15). Durante el mes de octubre de 2001, correspondiente al periodo de vaciante del río, la concentración de mercurio en el río Nanay fue de $<20$ a 230 ng/ $\mathrm{L}$, con promedio de $120 \mathrm{ng} / \mathrm{L}(\mathrm{n}=15)$. Las mayores concentraciones de mercurio en el río Nanay se encuentran cerca de la ciudad de Iquitos (figura 1). 


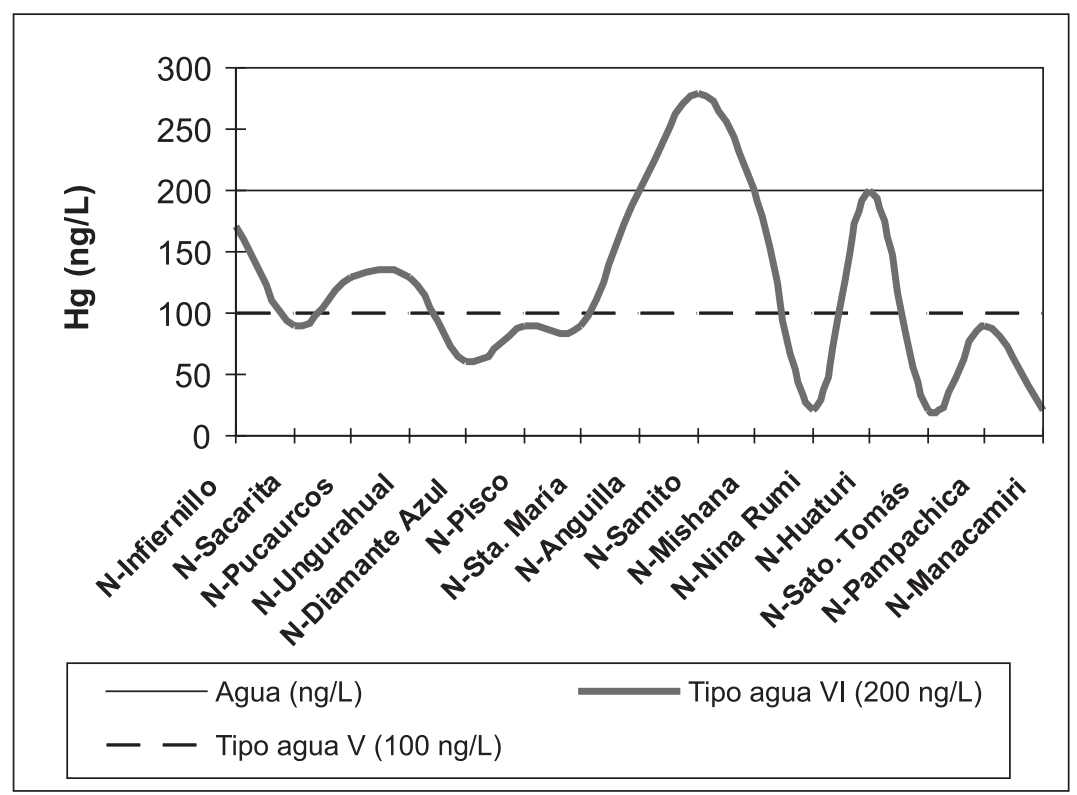

Nótese que algunas muestras de agua sobrepasan los límites máximos permisibles para los tipos de agua Vy VI de la Ley general de Aguas del Perú.

Figura 3. Contenido de mercurio en agua (mg/L) del río Nanay en el mes de octubre del 2001. Fuente: DIGESA

\section{Mercurio en sedimentos del fondo del río}

En las muestras de sedimentos del fondo del río Nanay colectadas en octubre de 2001, los niveles de mercurio oscilaron entre 0.005 a 0.0815 ìg/g, con un promedio de 0.0249 ìg/g; observándose un incremento de la concentración de mercurio río abajo como se muestra en la figura 4. Estos valores de mercurio no sobrepasan los límites máximos permisibles de 0.2 ìg/g, establecidos por la EPA. Las leyes peruanas no contemplan límites máximos permisibles de mercurio para este componente. 


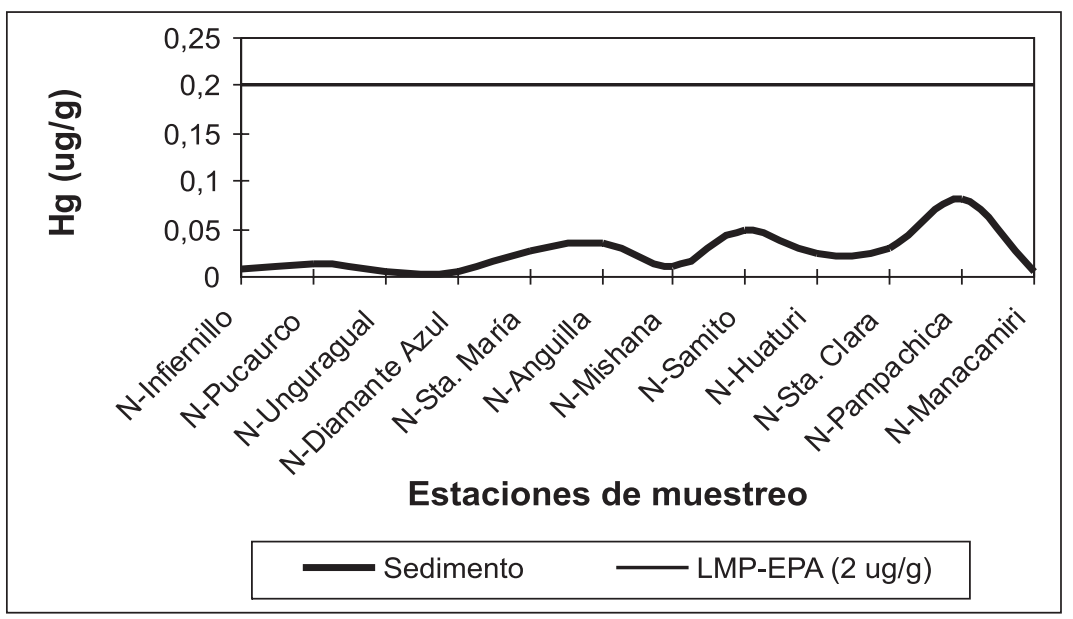

Las muestras de sedimentos no sobrepasan el límite máximo permisible (LMP) establecidos por Ia EPA.

Figura 4. Contenido de mercurio en sedimento de fondo del río Nanay (ìg/g) en el mes de octubre de 2001. Fuente: DIGESA

\section{Relación sedimento-agua}

Con la finalidad de tratar de explicar la presencia de mercurio en el agua se ha establecido una relación entre los niveles de mercurio encontrados en los sedimentos y en el agua del río Nanay (figura 5). Se observa que la concentración de mercurio en los sedimentos explican parcialmente, la presencia de las concentraciones de mercurio encontradas en el agua $(\mathrm{r}=0.158)$. En este contexto, el mercurio encontrado en el agua, además de provenir de los sedimento del río, podría provenir de otras fuentes.

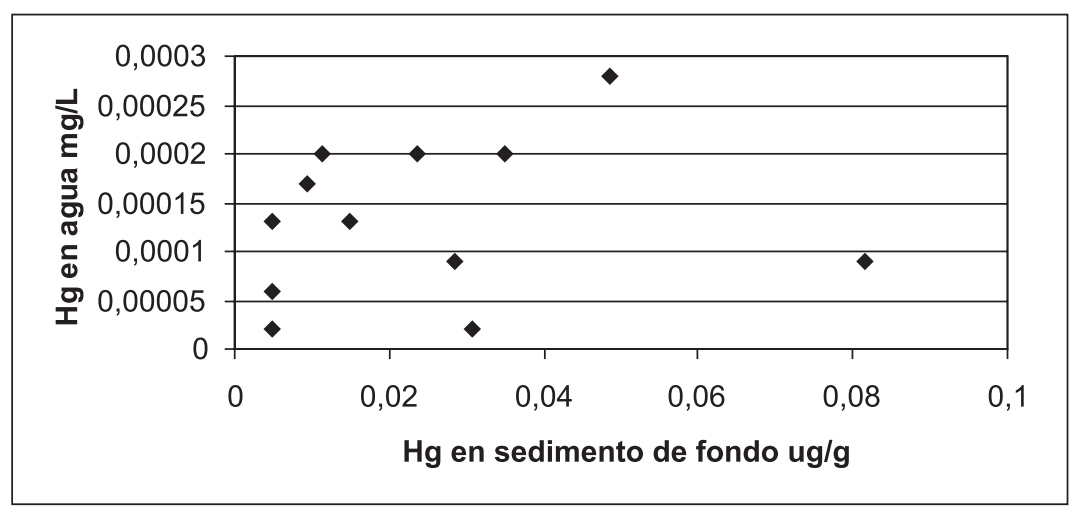

Nótese que algunas muestras de agua sobrepasan los límites máximos permisibles para los tipos de agua Vy VI de la Ley General de Aguas.

Figura 5. Relación de los contenidos de mercurio en agua (mg/L) y en los sedimentos (ìg/g) en el río Nanay durante el mes de octubre de 2001.

Fuente: DIGESA 


\section{DISCUSIÓN}

Los niveles de mercurio encontrados en el río Nanay son superiores a los valores de mercurio reportados para otros ríos de la Amazonía, como por ejemplo los ríos de agua blanca como el río Madre de Dios, Perú (Deza 1996) y el río Beni, Bolivia (Maurice-Bourgoin et al. 1999), en los que se realiza una importante actividad minera. Concentraciones de mercurio menores a las del río Nanay han sido encontrados también en el río Amazonas, en territorio brasileño (Roulet et al. 2001b). En estos ríos de agua blanca las mayores concentraciones de mercurio se encuentran en las partículas en suspensión (Roulet et al. 2001b). En el río Madre de Dios, estas partículas en suspensión compuestas mayormente de arena, arcilla y limo proporcionan al agua una coloración marrón y escasa transparencia de la columna de agua de alrededor de 16 a 24 cm (IIAP-CTAR Madre de Dios 2000). En cambio, el río Nanay presenta características limnológicas de agua negra con buena transparencia debido a que las partículas en suspensión son escasas (IIAP 1999a; 1999b; 2002) de ahí que la mayor concentración de mercurio se encuentre en el agua.

Las altas concentraciones de mercurio en el río Nanay también pueden estar relacionadas con la magnitud de su caudal. El Nanay presenta un caudal muy reducido (alrededor de 240 m³/s; IIAP 1999a; 1999b; 2002) con relación a los ríos de agua blanca anteriormente descritos, como por ejemplo, el río Madre de Dios, que en el lado peruano presenta un caudal de 9,281 m³/s (IIAP-CTAR Madre de Dios 2000).

De acuerdo a la legislación peruana, en el río Nanay existen algunos lugares donde se presentan niveles de mercurio superiores a los límites máximos permisibles para dos tipos de uso de aguas (El Peruano 1983). Los niveles de mercurio en el río Nanay no permitirían realizar el aprovechamiento de algunos recursos hidrobiológicos, tal es el caso de los moluscos bivalvos para los cuales se ha establecido que las zonas de pesca de estos moluscos no debe de sobrepasar los $100 \mathrm{ng} / \mathrm{L}$ de mercurio (agua de tipo de uso V). En este contexto, la mayoría de los lugares muestreados presentan niveles de mercurio que sobrepasan el nivel antes señalado (figura 3). En el río Nanay existen algunos lugares de muestreo donde los niveles de mercurio sobrepasan los $200 \mathrm{ng} / \mathrm{L}$ de mercurio, nivel que es establecido como límite máximo permisible para las zonas de preservación de fauna acuática y pesca recreativa o comercial (agua de tipo de uso VI, figura 3).

Para otros usos del agua del río Nanay, como el abastecimiento de agua potable para la ciudad de Iquitos, los niveles de mercurio son muy bajos y, aparentemente, no son perjudiciales para la población iquiteña. Sin embargo, más de 25,000 habitantes de las poblaciones ribereñas consumen diariamente el agua en forma directa, sin ningún tratamiento de potabilización. En este marco, se calcula una ingesta diaria promedio de mercurio de 0.36 ìg/L y mensual de 10.8 ìg/L, considerando, por un lado, la ingesta diaria de tres litros de agua por habitante como promedio, ya para calmar la sed con agua, refresco u otro o de agua usada para la preparación de los alimentos.

Con relación a los sedimentos del fondo del río Nanay, las concentraciones de mercurio son inferiores a las encontradas para otros ambientes acuáticos de la Amazonía, como por ejemplo para el río Madre de Dios, Perú (Mora 1995, citado en Deza 1996) y para el río Madeira, Brasil (Malm et al. 1995 y Martinelli et al. 1988, citados en Deza 1996). Este hecho corrobora la tesis de que en la Amazonía se ha identificado como una de las fuentes de mercurio a los sedimentos acarreados de las laderas de los Andes (Maurice-Bourgoin et al. 1999; Roulet et al. 2001a). Este es el caso de los ríos de agua blanca mencionados anteriormente, pero no lo es para el río Nanay, que tiene origen en el llano amazónico y los sedimentos escasos que acarrea son provenientes del bosque de tierra firme, de las zonas hidromórficas y de las áreas que inunda periódicamente durante el periodo de creciente.

Considerando las concentraciones de mercurio en los sedimentos y las cantidades del mismo que son removidas por dragas (600 m³/día, de acuerdo a los estudios de impacto ambiental AUDITEC SAC-DINAMICA 2000), se realizaron cálculos sobre liberación de mercurio provenientes de los sedimentos del fondo del río. Si consideramos la concentración promedio de 0.0249 ìg/g de mercurio en el sedimento, una draga estaría removiendo 1,328 mg de mercurio en los ocho meses de trabajo al año en los que el nivel de las aguas es adecuado para la realización de esa actividad. 


\section{CONCLUSIONES}

Los niveles promedio del contenido de mercurio en el río Nanay se incrementan de <20 ng/L en el 2000 a 120 ng/ L en el 2001. En este río se registran concentraciones de mercurio superiores a los límites máximos permisibles para los tipos de agua V (aguas de zonas de pesca de mariscos bivalvos) y VI (aguas de zonas de preservación de fauna acuática y pesca recreativa o comercial) establecidos en las leyes peruanas (El Peruano 1983). En los sedimentos de fondo se presentan concentraciones de mercurio que oscilan entre 0.005 a $0.0815 \mathrm{~g} g / \mathrm{g}$, valores que se mantienen por debajo de los límites máximos permisibles establecidos por la EPA. No obstante, se infiere una importe fuente de mercurio proporcionado por la actividad minera que se desarrolla en la cuenca, la misma que realiza una fuerte remoción de sedimentos del fondo del río, afectando a diferentes nichos ecológicos, en perjuicio directo de las especies bentónicas.

En este contexto, se puede predecir que la continuación e incremento de las actividades de extracción minera, especialmente la aurífera, en la cuenca del río Nanay, producirá mayores impactos en perjuicio de la conservación de la cuenca y el uso sostenible de sus recursos naturales.

\section{RECONOCIMIENTO}

A las instituciones integrantes de la Comisión Técnica Multisectorial para la evaluación de las actividades auríferas en el río Nanay por su participación en los periodos de muestreo; al IIAP y a la Comisión Ambiental de Loreto por el apoyo decidido para la realización del estudio y a la Dirección General de Salud Ambiental-DIGESA, por la realización de los análisis de mercurio en agua y sedimentos del fondo del río.

\section{BIBLIOGRAFÍA}

AUDITEC SAC-DINAMICA. 2000. Estudio de Impacto Ambiental: Dragado en el río Nanay. Dinámica Minera y Agroindustrial S.A.-DIMIASA. Ministerio de Energía y Minas Dirección General de Asuntos Ambientales. Loreto-Perú.

CARMOUZE, J.P., M. LUCOTTE AND A. BOUDOU. 2001. Mercury en the Amazon: Importance of human and environment, health hazards. IRD Editions. París. 40 pp.

CLARKSON, T.W. 2002. The three modern faces of mercury. Environmental Health Perspectives, 110(1): 11-22.

DEZA, N.E. 1996. Mercury acumulation in fish from Madre de Dios, a goldminig area in the Amazon basin, Perú. Thesis of Master of Science. Oregon. 39 p.

EL PERUANO. 1983. Decreto Supremo n. 007-83-SA. 17 de marzo de 1983: 14698-14700.

ESPAÑOL, C. S. 2001. Toxicología del mercurio. Actuaciones preventivas en sanidad laboral y ambiental. CD: Jornada internacional sobre impacto ambiental del mercurio utilizado por la minería aurífera artesanal en Iberoamérica. Setiembre de 2001. Lima-Perú. 69 p.

FAO 1983. Compilation of legal limits for Hazardous Sunstances in Fish and Fishery Products. FAO. Fisheries Circular n. ${ }^{\circ}$ 764. Roma.

GÓMEZ, R. 1994. Contaminación ambiental en la Amazonía peruana. Reporte 1994. Informe técnico de avance. Instituto de Investigaciones de la Amazonía Peruana. Iquitos-Perú. 52 p. + anexos. 
GOLDMAN, L. R., M.W. SHANNONAND THE COMMITTEE ON ENVIRONMENTAL HEALTH. 2001. Technical Report: Mercury in the Environment: Implications for Pediatricians. American Academy of Pediatrics. PEDIATRICS, 108(1): 197-205.

GRADE, 1994. Estudio de la minería informal y el medio ambiente (Sub Proyecto MIMA) Zona: Madre de Dios. Informe final. Programa de Asistencia Técnica al Sector Energía y Minas. 135 pp.

IIAP (Instituto de Investigaciones de la Amazonía Peruana). 1999a. Caracterización Física de la cuenca del río Nanay. Proyecto Integral y Sostenible del río Nanay de la Amazonía Peruana. Programa de Ordenamiento Ambiental. Iquitos. 14 pp.

IIAP (Instituto de Investigaciones de la Amazonía Peruana). 1999b. Informe final proyecto «Evaluación de la fauna silvestre en el área de influencia de la carretera Iquitos - Nauta» y propuesta de un plan de manejo de la fauna silvestre. Iquitos. 78 pp.

IIAP (Instituto de Investigaciones de la Amazonía Peruana). 2000a. Evaluación y conservación de la diversidad biológica para el ecoturismo. Informe Final del Proyecto. Iquitos.92 p.

IIAP (Instituto de Investigaciones de la Amazonía Peruana). 2000b. Caracterización de ecosistemas para protección y uso de la cuenca del río Nanay. Conservación y uso de ecosistemas. Informe Anual. 62 p.

IIAP (Instituto de Investigaciones de la Amazonía Peruana). 2002. Propuesta de Zonificación Ecológica Económica de la cuenca del río Nanay. Programa de Ordenamiento Ambiental-Proyecto Conservación de la Biodiversidad y Manejo Comunitario de los Recursos Naturales de la Cuenca del Río Nanay. Convenio IIAP-Banco Mundial. Tipog. 111 p.

IIAP (Instituto de Investigaciones de la Amazonía Peruana)-CTAR Madre de Dios (Consejo Transitorio de Administración Regional de Madre de Dios). 2000. Medio físico, zonificación ecológica económica de la región Madre de Dios. Volumen II, Puerto Maldonado, 151 p.

IMA (Instituto de Manejo de Agua y Medio Ambiente). 1995. Efectos de la contaminación por mercurio en la explotación de oro en Madre de Dios. Cusco. 45 p.

KALES, S.N. AND R.H. GOLDMAN. 2002. Mercury Exposure: Current concepts, controversies and a Clinic's experience. Jornal of Ocupational and Environmental Medicine, 44(2).

LACERDA, L.D. 1995. Amazon mercury emissions. Nature, 374: 20-21.

LODENIUS, M. AND O. MALM. 1998. Mercury in the Amazon. Rev. Environ. Contam. Toxicol, 157: 25-52.

LUNDBERG, B. 1999. Actividad aurífera y medio ambiente: con comentarios sobre el trabajo de zonificación. Programa de zonificación ecológica económica para el desarrollo sostenible de la cuenca del río Madre de Dios. Tipog. 16 p.

MAURICE-BOURGOIN, L., I. QUIROGA, J.L. GUYOTAND O. MALM. 1999. Mercury pollution in the upper Beni River, Amazonian Basin: Bolivia. Ambio 28 (4):302-306.

REYES, F.C. 2000. Explotación de oro aluvial en el río Nanay. Informe s/n-2000-CTAR-L-ST/CRF. Tipog. 20 pp.

ROULET, M., J. R. D. GUIMARÃES AND M. LUCOTTE. 2001. Methylmercury production and accumulation in sediments and soils of Amazonian floodplain - Effect seasonal inundation. Water, Air, and Soil Pollution, 128: 41-60. 
ROULET, M., M. LUCOTTE, R. CANUEL, N. FARELLA, M. COURCELLES, J.R.D. GUIMARÃES, D. MERGLER AND M. AMORIM. 2000a. Increase in mercury contamination recorded in lacustrine sediments following deforestation in the central Amazon. Chemical Geology, 165: 243-266.

ROULET, M., M. LUCOTTE, R. CANUEL, N. FARELLA, G. Y. G. DE FREITOS, P. J. R. PACHECO, J.R.D. GUIMARÃES, D. MERGLER AND M. AMORIM, 2001b. Spatio-temporal geochemistry of mercury in waters of the Tapajós and Amazon rivers, Brazil. Limnol. Oceanogr., 46(5): 1141-1157.

ROULET, M., M. LUCOTTE, N. FARELLA, G. SERIQUE, H. COELHO, C.J. SOUSA PASSOS, E. DE JESÚS DA SILVA, P. SCAVONE DE ANDRADE, D. MERGLER, J. R. D. GUIMARÃES AND M. AMORIM. 1999. Effects of recent human colonization on the presence of mercury in Amazonian ecosystems. Water, Air, and Soil Pollution, 112: 297-313.

ROULET, M.; M. LUCOTTE, J. R. D. GUIMARÃES AND I. RHEAULT. 2000b. Methylmercury in water, seston and epiphyton of an Amazonian river and its floodplain, Tapajós River, Brazil. The Science of the Total Environment 261: 43-59.

SIOLI, H. (ed.). 1984. The Amazon. Linmology and landscape ecology of a mighthy tropical river and its. Dr. Junk Publishers, Dordrecht. 763 p.

VEIGA, M.M. 1997a. Introducing new technologies for abatement of global mercury pollution in Latin America. Rio de Janeiro: UNIDO/UBC/CETEM/CNPq. 64 p.

VEIGA, M.M. 1997b. Mercury in artisanal gold mining in Latin Amarica: facts, fantasies and solutions. UNIDOExpert Group Meeting-Introducting new technologies for abatement of global mercury pollution deriving from artisanal gold mining. Vienna. 23 p. 